\title{
Subtypes of brain activation are heritable and genetically linked with behavior in the Human Connectome Project sample
}

Benhajali Yassine ${ }^{1,2}$, Badhwar AmanPreet ${ }^{2}$, Urchs Sebastian ${ }^{2,4}$, Moreau Clara ${ }^{5,2}$, Chouinard-Decorte Francois $^{4}$, Vainik Uku ${ }^{3,4}$, Ferre Perrine ${ }^{2}$, Orban Pierre ${ }^{6,7}$, Pérusse Daniel ${ }^{1}$, Bellec Pierre ${ }^{2,8}$

${ }^{1}$ Département d'anthropologie, Université de Montréal, Montréal, Québec, Canada.

${ }^{2}$ Centre de recherche de l'Institut universitaire de gériatrie de Montréal (CRIUGM), Montréal, Québec, Canada.

${ }^{3}$ Institute of Psychology, University of Tartu, Estonia.

${ }^{4}$ Montreal Neurological Institute, Montréal, Québec, Canada.

${ }^{5} \mathrm{CHU}$ Sainte-Justine Research Center, Montréal, Québec, Canada.

${ }^{6}$ Département de Psychiatrie et d'addictologie, Université de Montréal, Montréal, Québec, Canada.

${ }^{7}$ Centre de recherche de l'Institut universitaire en santé mentale de Montréal

${ }^{8}$ Département de psychologie, Université de Montréal, Montréal, Québec, Canada.

\section{Abstract}

Many imaging and genetics studies have aimed to clarify whether the brain acts as an intermediate phenotype mediating the influence of genes in human behaviour. Brain activations in response to task demands are heterogeneous at the individual level, but also follow common patterns at the group level. Some studies have addressed this tension between heterogeneity and homogeneity by identifying groups of individuals that share the same brain activations patterns, called brain activation subtypes. In this work, we aimed to assess the viability of brain subtypes as endophenotypes intermediate between genes and behavior. We extracted brain activation subtypes separately for seven fMRI tasks, in 842 participants from the Human Connectome Project (HCP). We estimated the heritability of these subtypes and their genetic correlation with behavioral measures obtained inside and outside the scanner. Across all tasks, subtypes ranged from a predominantly 'deactivating' pattern towards a more 'activating' pattern of brain activity, with a heritability estimate ranging from 0 to 0.62 . We observed high genetic and phenotypic correlation between behavioral measures and brain activation subtypes only for language and working memory tasks. Our results showed a significant genetic grounding of brain activation subtypes and they appear as a simple yet effective technique to tackle heterogeneity into imaging genetics studies.

Keywords: Task-fMRI, Subtypes, Heritability, Genetic Correlation, Behavioral measures. 


\section{Introduction}

Human actions and interactions are influenced by a hierarchy of factors such as genetic make-up, environmental influences, culture, individual values and attitudes. Within this hierarchical system, functional brain organization is an intermediate phenotype, and could be key to understanding how factors of nature and nurture drive variations in human behavior. Activation maps generated using non-invasive functional magnetic resonance imaging (fMRI) offer a window into the individual functional organization for a specific cognitive context. It is well established that these activation maps exhibit substantial variability across individuals (Van Horn et al., 2008), which may explain inter-individual differences in observable behaviour. However, it is still unclear how this functional brain variability relates to behavioral differences in terms of genetic and environmental influences. The main objectives of the present study were to (1) estimate the heritability of brain activation subtypes, i.e. groups of individuals who reliably share similar activation maps and, (2) to assess whether these subtypes share their genetic basis with behavioural measures across a broad range of cognitive tasks.

\section{Inter-individual differences in brain activation}

Task fMRI activation maps exhibit substantial inter-individual differences that likely reflect both fundamental differences in the anatomo-functional organization of brain networks, and differences in cognitive strategies. An early electrophysiological study comparing proficient and poor readers showed that proficient readers had more efficient neural processes, as reflected by lower power spectra in EEG (Maxwell et al., 1974). In a more recent fMRI study using a sentence-picture verification paradigm, Miller and colleagues (2012) manipulated the use of either verbal or visual strategies in a memory retrieval task in healthy subjects. They found that larger differences between two individuals in their tendency to visualize highly imageable word stimuli were associated with larger differences between their patterns of brain activity across the whole brain. Differences in brain activation related to either cognitive strategies or individual abilities have been demonstrated across a wide range of cognitive domains (Feredoes \& Postle, 2007; Heun et al., 2000; Machielsen et al., 2000; McGonigle et al., 2000; Miller et al., 2002, 2009; Parasuraman \& Jiang, 2012; Seghier et al., 2008; Seghier \& Price, 2009). Such inter-individual differences in brain activation have not only been observed for complex cognitive processes but even for simple tasks. For example, Seghier and colleagues (2016) found that the primary motor cortex was missing from the group activation map in a motor task, but was visible in nearly $50 \%$ of individual activation maps. Taken together, these observations show that average group activation maps do not accurately represent the neural systems engaged by the individuals that make up that group (Lebreton \& Palminteri, 2016). 


\section{Brain subtypes}

Clustering strategies such as hierarchical clustering have the potential to study the inter-individual heterogeneity in brain activation. A cluster analysis summarizes the heterogeneity of brain maps using a finite number of subtypes (See Figure 1). Hierarchical clustering was for instance applied to aggregate subjects based on the similarity of their brain activation maps (Kherif et al., 2003), and to identify atypical subjects (Seghier et al., 2007). Clustering-based subtyping has recently gained popularity for the dissection of brain heterogeneity, for example the delineation of cortical atrophy subtypes in Alzheimer's disease (Badhwar, McFall, et al., 2019), and resting-state connectivity subtypes in the autism spectrum (Easson et al., 2019; Urchs et al., 2020). In the non-clinical domain, some studies have linked brain functional subtypes with normal behavioral and demographic measures. A recent study by Kashyap and colleagues (2019) investigated resting-state connectivity subtypes of 788 healthy subjects from Human Connectome Project (HCP). They estimated the common component of each subject from the four resting states runs, and classified the subjects based on the similarity/dissimilarity of their components. Two subgroups emerged, one with high similarity and another with high dissimilarity between components. Both subgroups associated with behavioral measures related to the use of marijuana, illicit drugs, alcohol, and tobacco, and antisocial personality. Another study by Smith and colleagues (2015) showed that a positive-negative mode of population covariation links brain connectivity, demographics, and behavior. Finally, a study by Kirchhoff and Buckner (2006) reported that different cognitive strategies (visual vs verbal) used by subjects in the scanner to perform a memory task were correlated with distinct brain activation networks. They also found that activity in brain regions associated with the use of these strategies was also correlated with memory performance. These studies demonstrate that heterogeneity in brain function exists and may be characterized as subtypes using unsupervised data mining tools.

Despite the growing literature on resting-state-based brain subtyping, only few studies explored the potential use of task-based brain subtyping (activation subtpes). Therefore, it is unclear if activation subtypes could serve as a relevant intermediate phenotype between genes and behaviour. In particular, are brain activations subtypes genetically driven? And, if yes, are the same genes driving inter-individual variations in behaviour and brain subtypes?

\section{Genetic of brain activation subtypes}

A quantitative phenotype, or trait, depends on the cumulative actions of many genes and of the environment. The quantitative genetic analysis uses either family-based quantitative data or twins-based data to partition the observed covariance among related individuals into genetic and environmental components. Heritability is defined as the contribution of additive genetic variance to the 
total variance of a trait (Falconer \& Mackay, 1996) and can be estimated for any quantitative phenotype. A meta-analysis from imaging genetics showed that variations in neuroimaging phenotypes, and in particular activation maps, are substantially (ex: up to $0.75 \%$ on visuomotor task) explained by additive genetic factors (Blokland et al., 2012). Current methods in quantitative genetics to estimate the heritability of fMRI activation maps, however suffer from important limitations. The large number of voxel- and vertex-wise measurements in imaging genetics make heritability estimates very challenging in terms of computational load and the need to account for multiple testing (Ganjgahi et al., 2015). Studies that estimated heritability of brain activations tended to mitigate multiple comparisons by targeting regions of interest (Babajani-Feremi, 2017; Blokland et al., 2008, 2011, 2017; Chen et al., 2018; Pinel \& Dehaene, 2013; Rao et al., 2018; van der Meulen et al., 2018). To our knowledge, there is no study that investigated heritability of brain activation subtypes in healthy samples. Investigations of the heritability of brain activation maps will thus benefit from the subtyping approaches reviewed earlier, as they provide compact summaries of the variations in brain activity across subjects, making it easier to interpret what specific characteristics of activation maps are under genetic control.

Some studies attempted to estimate whether brain activation maps and behavioural measures are influenced by similar groups of genes. This concept is called pleiotropy or genetic correlation between phenotypes. Le Guen and colleagues (2018) used HCP data to perform bivariate genetic analyses between the fMRI activation maps and fluid intelligence, as well as the performance of subjects on working memory and language domain tasks. Regions of the language network along the superior temporal sulcus and the angular gyrus were significantly genetically correlated with indicators of cognitive performance (Fluid intelligence, working memory, vocabulary comprehension and reading decoding). Rao and colleagues (2018) also provided evidence for the genetic correlation between risk-taking behavior and risk-related brain activation. Those studies used a voxel-level analysis on regions of interest in order to estimate the genetic correlation between brain phenotypes and behavioral phenotypes. In this work we used subtypes weights as brain phenotype to capture genetic link with behavioral measures.

\section{Aims and hypotheses}

In this work, we aimed to demonstrate the viability of activation subtypes as an intermediate endophenotype between genes and behaviour. We implemented a clustering approach to identify subtypes of brain activation in the fMRI tasks of the HCP sample. Our two main objectives were 1) to test the heritability of these brain activation subtypes 2) to test the association and shared genetic variance between brain activation subtypes and a battery of behavioural measures. The clustering technique proceeded by aggregating individual brain activation maps into a limited number of subtypes. The similarity between each individual map and each subtype was then estimated with a continuous subtype weight. Using these subtype weights as a trait, we specifically aimed to: 
1- Test the association of subtypes weights with behavioral measures, collected outside of the scanner, as well as in-scanner task performance. Our hypothesis was that the performance of each task would be associated mainly with the brain activation subtypes derived from this task, while behavioural measures would be associated with tasks drawn from a related cognitive domain.

2- Evaluate if these activation subtypes are heritable, using a twin family design. Our hypothesis was that subtype of brain activation would show moderate to high heritability

3- Quantify the genetic correlation between subtypes of brain activation and behavioral measures in several domains, as well as with in-scanner task performance. Our hypothesis was that these genetic correlations would be moderate to high between behavioral and brain phenotypes that were related to similar cognitive abilities.

\section{Methods}

\section{Subjects}

We used the S900 data release from the HCP open access database (Van Essen et al., 2013). The S900 release includes behavioral and imaging data from 897 healthy adult participants collected August 2012-March 2015, out of which 862 healthy adults (aged 22-35 y) successfully completed at least one of the $7 \mathrm{fMRI}$ tasks. Post quality control, 842 subjects were selected for the analysis included 278 families (742 individuals), and 100 unrelated subjects. Families were either monozygotic twins (genetically identical, 84 pairs, 168 individuals) or dizygotic/fraternal twins (genetically no more related than ordinary full siblings, 77 pairs, 154 individuals) and 89 families of non-twin siblings (209 individuals) (Van Essen et al., 2013).

The HCP sample reflects the ethnic diversity of American families (White non-Hispanic, Hispanic, Asian and African-American). HCP provides the required ethics and consent needed for study and dissemination. This study went through further internal institutional data analysis approval.

\section{Imaging data}

MRI data for the S900 release was collected on a customized Siemens MAGNETOM Connectom 3T scanner at Washington University. The data is composed of multiple imaging sessions covering four modalities: structural (T1w and T2w), resting-state fMRI, task fMRI (tfMRI, 7 tasks) and diffusion MRI (dMRI) (Hodge et al., 2016). In this work we only used T1w and tfMRI modalities. For T1w images a 32 min was spent acquiring each MPRAGE image. An MPRAGE (Mugler \& Brookeman, 1990) sequence with $0.7 \mathrm{~mm}$ isotropic resolution (FOV $=224 \mathrm{~mm}$, matrix $=320,256$ sagittal slices in a single slab), TR $=2400 \mathrm{~ms}, \mathrm{TE}=2.14 \mathrm{~ms}, \mathrm{TI}=1000 \mathrm{~ms}, \mathrm{FA}=8^{\circ}$, Bandwidth $(\mathrm{BW})=210 \mathrm{~Hz}$ per pixel, Echo Spacing 
$(E S)=7.6 \mathrm{~ms}$ (see (Glasser et al., 2013) for more detail). For each subject and task, 2 runs of tfMRI time series data were collected, 1 run in each phase encoding direction, left-to-right (LR) then right-to-left $(\mathrm{RL})$, with a temporal resolution of $0.73 \mathrm{~s}$ and a spatial resolution of 2-mm isotropic, using a standard 32-channel Siemens coil (Van Essen et al., 2013)

All participants included completed 7 tasks in the MRI scanner - Working Memory (405 frames/scan), Gambling (253 frames/scan), Motor (284 frames/scan), Language (316 frames/scan), Social Cognition (274 frames/scan), Relational Processing (232 frames/scan), and Emotion Processing (176 frames/scan) (Hodge et al., 2016).

\section{Image preprocessing}

From HCP S900 data release, we used the unprocessed tfMRI data. Each subject was preprocessed using the Neurolmaging Analysis Kit version dev-0.14.0 (NIAK) ${ }^{1}$, under CentOS with Octave ${ }^{2}$ version 3.8.1 and the Minc toolkit ${ }^{3}$ version 0.3.18. Analyses were executed in a high performance computing environment $^{4}$, using the pipeline system for Octave and Matlab (PSOM) (Bellec et al., 2012).

Each tfMRI image was corrected for differences in timing of slice acquisitions; a rigid-body motion was then estimated using Minctracc (Collins \& Evans, 1997) for each time frame, both within and between runs, as well as between one fMRI run and the T1 scan for each subject. The T1 scan was itself non-linearly co-registered to the Montreal Neurological Institute (MNI) ICBM152 stereotaxic symmetric template (Fonov et al., 2011), using the CIVET pipeline (Ad-Dab'bagh et al., 2006). The rigid-body, fMRI-to-T1 and T1-to-stereotaxic transformations were all combined to resample the fMRI in MNI space at a $3 \mathrm{~mm}$ isotropic resolution. To minimize artifacts due to excessive motion, all time frames showing an average frame displacement (FD) greater than $0.5 \mathrm{~mm}$ were removed (Power et al., 2012). The following nuisance covariates were regressed out from the tfMRI time series: slow time drifts (basis of discrete cosines with a $0.01 \mathrm{~Hz}$ high-pass cut-off), average signals in conservative masks of the white matter and the lateral ventricles as well as the first principal components (accounting for 95\% variance) of the six rigid-body motion parameters and their squares (Giove et al., 2009). Finally, the tfMRI volumes were spatially smoothed with a $6 \mathrm{~mm}$ isotropic Gaussian blurring kernel. A more detailed description of the pipeline can be found on the NIAK website ${ }^{5}$.

\footnotetext{
${ }^{1}$ http://niak.simexp-lab.org/

2 https://www.gnu.org/software/octave/

3 http://bic-mni.github.io/

4 https://www.calculquebec.ca/

5 http://niak.simexp-lab.org/build/html/PREPROCESSING.html
} 


\section{Registration and motion quality control}

We used a structured visual quality control procedure (Benhajali et al., 2020) to assess the registration between the individual T1 scans and the stereotaxic template space, as well as registration between the individual T1 and the median functional image. The quality control procedure included a series of key anatomical landmarks and associated confidence intervals. The quality control procedure is publicly available as a tutorial on the Zooniverse platform (Brain Match, 2015). Subjects were excluded from the analysis in cases of failed coregistration with the template space or where less than 100 time points had acceptable amounts of head motion after scrubbing (see next section). A total of 843 subjects (477 female) passed quality control criteria for inclusion in the analysis.

In scanner head motion is a critical confounding factor in functional MRI and tends to act as a relatively stable, heritable phenotype (Couvy-Duchesne et al., 2014, 2016; Engelhardt et al., 2017). Removing time points affected by large head motion, a technique known as scrubbing, is efficient at reducing the effect of head motion on fMRI time series (Caballero-Gaudes \& Reynolds, 2017). Average framewise displacement (FD) (Power et al., 2012) is a summary measure of head displacement throughout an fMRI acquisition, and can be estimated both before and after scrubbing. We assessed the heritability of average FD motion measure before and after removal of time frames with excessive motion (time frames with FD greater than $0.5 \mathrm{~mm}$ ). Heritability analysis was implemented using the SOLAR Eclipse software, as described in the dedicated Methods section below. Figure Sup1 (supplementary material) shows that FD measures before scrubbing had substantial (and significant) heritability estimates, ranging from 0.22 to 0.52 across tasks, which is consistent with previous reports (Couvy-Duchesne et al., 2014, 2016; Engelhardt et al., 2017). After scrubbing, the heritability estimates of FD ranged from 0 to 0.13 across tasks. We concluded that using scrubbing as a preprocessing step was beneficial for further heritability analysis of fMRI activation maps, as it reduced the potential confounding effects of heritable motion artifacts.

\section{Task fMRI}

\section{Working Memory}

$\mathrm{N}$-back tasks were used as follows: the participant was presented with a series of pictures, drawn from different categories (faces, tools, places and body parts). Half of the blocks were used for a 2-back memory trial, where the participant was asked to respond each time whether the current stimulus was the same as the one from two back. The other half of the blocks were used for a 0-back working memory task, where the individual had to recognize a single 'target' for any stimulus during the block. 
Participants played a game of card guessing, and were asked to guess the number on a mystery card (question mark card) to win or lose money. The potential number on the mystery card was an integer between 1-9. Pressing one of two buttons, the participants had to guess if the number was over or below 5 . A successful guess was rewarded by " $\$ 1$," the participants lost $-\$ 0.50$ for bad guesses, and nothing for neutral guesses (where the number was exactly 5 ). The task was presented in blocks of 8 trials that were either mostly reward (6 reward trials pseudo randomly interleaved with either 1 neutral and 1 loss trial, 2 neutral trials, or 2 loss trials) or mostly loss (6 loss trials interleaved with either 1 neutral and 1 reward trial, 2 neutral trials, or 2 reward trials). In each of the two runs, there were 2 mostly reward and 2 mostly loss blocks, interleaved with 4 fixation blocks ( $15 \mathrm{~s}$ each)

\section{Motor}

Participants were presented with visual cues that asked them to tap their left or right fingers, squeeze their left or right toes, or move their tongue, in order to generate maps of different motor areas. Each block of a movement type lasted $12 \mathrm{~s}$ (10 movements), and was preceded by a 3 seconds cue. In each of the two runs, there were 13 blocks, with 2 blocks of each body part and three blocks of $15 \mathrm{~s}$ fixation.

\section{Language Processing}

The task consisted of two runs that each interleave 4 blocks of a story condition and 4 blocks of a math condition. The task was designed so that the math blocks match the length of the story blocks, without fixation between block transitions. The story blocks presented participants with brief auditory stories (5-9 sentences) adapted from Aesop's fables, followed by asking participants to select the topic of the story amongst two alternatives (button press response). The math blocks required subjects to complete addition and subtraction problems (auditory instructions, and select the right answer amongst two alternatives (button press response).

\section{Social Cognition (Theory of Mind)}

Participants were presented with short video clips (20 s) of objects (squares, circles, triangles) either interacting in some way, or moving randomly. Participants were asked to judge whether a social or a random interaction was happening between these objects on the video. Each of the random and social conditions had 5 video blocks ( 2 Mental and 3 Random in one run, 3 Mental and 2 Random in the other run) and 5 fixation blocks ( $15 \mathrm{~s}$ each).

\section{Relational Processing}

In the relational processing condition, participants were presented with 2 pairs of objects, with one pair at the top of the screen and the other pair at the bottom of the screen. They were told that they should 
first decide what dimension differed across the top pair of objects (shape or texture) and then they should decide whether the bottom pair of objects also differed along that same dimension.

\section{Emotion Processing}

Participants were presented with blocks of trials that either asked them to decide which of two faces presented on the bottom of the screen matched the face at the top of the screen, or which of two shapes presented at the bottom of the screen matched the shape at the top of the screen. The faces had either angry or fearful expressions.

For more details on tfMRI in the HCP, see Barch and colleagues (2013).

\section{Activation maps}

For each of the 7 tasks, individual activation maps were extracted from block design conditions using the statistical analysis toolbox FMRISTAT (Worsley et al., 2003). Different conditions were included in some of the tasks (e.g. foot, hand and tongue for the motor task), resulting in a total of 21 conditions. For each of the 7 tasks, the stimuli in the design matrices were convolved with a hemodynamic response function modeled as a difference of two gamma functions. Temporal drift was removed by adding a cubic spline in the frame times to the design matrix, and spatial drift was removed by adding a covariate in the whole volume average. Autocorrelation parameters were estimated at each voxel and used to whiten the data and design matrix.

\section{Subtypes of activation maps and weights extraction}

a

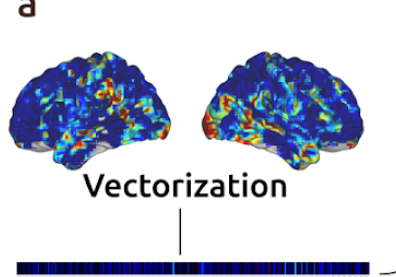

Voxels

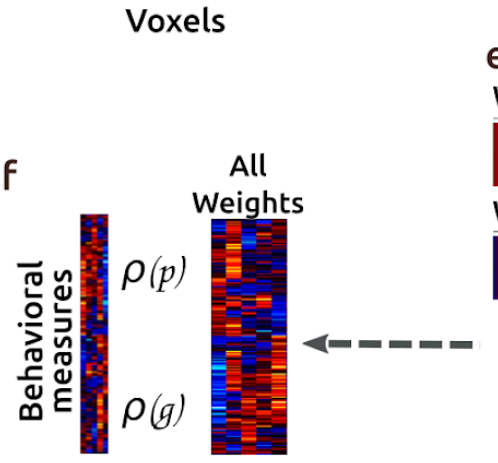

Voxels

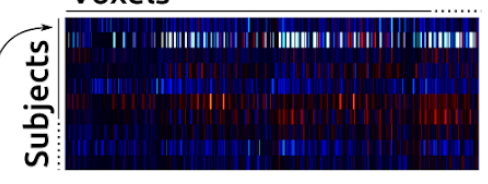

e

Weights Subtype 1

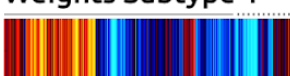

Weights Subtype 2
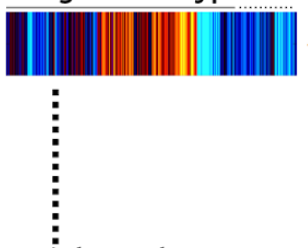

b

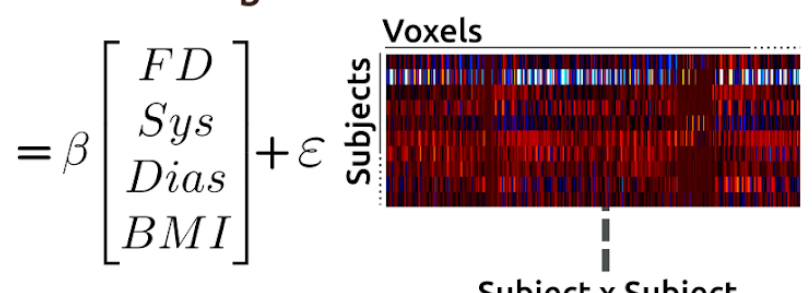

Subject x Subject

d

C Correlation

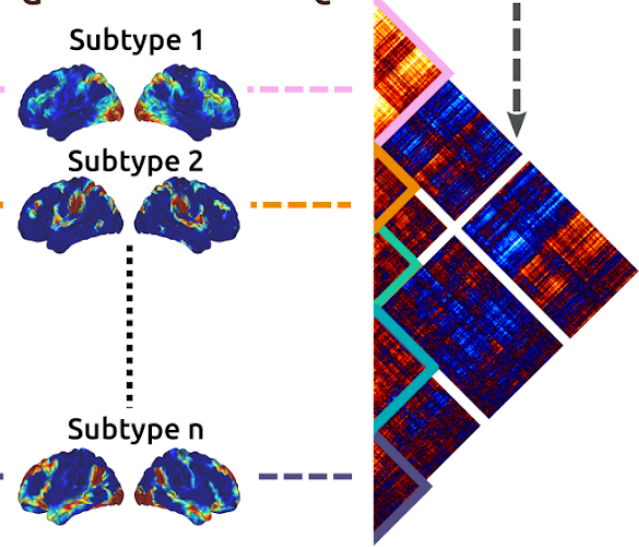


Figure 1 - Activation subtypes maps generation and weights extraction. a. From each subject, an un-thresholded statistical parametric map (SPM) is vectorized then stacked. b. Nuisance regression from stacked SPM (FD: Frame Displacement, Sys: Systolic blood pressure, Dias: Diastolic blood pressure, BMI: Body Mass Index). c. From the residual stack, a subject by subject correlation matrix is clustered into subgroups of activations maps (subtypes). d. Each subtype is represented spatially by a mean activation map of all subjects within the same cluster . e. Each subtype is represented on the subject level by its weight (correlation between a vectorized subject activation map and a vectorized subtype map). f. Individual weights are correlated genetically ( $\left.\rho_{\mathrm{g}}\right)$ and phenotypically $\left(\rho_{\mathrm{p}}\right)$ with behavioral measures.

For each of the 21 task conditions, a nuisance regression was applied on stacked activation maps (Figure 1a). Nuisance regressors were frame displacement (FD), systolic blood pressure, diastolic blood pressure (Sys Dias) and body mass index (BMI) (Figure 1b). Then, a subject by subject similarity (Pearson's correlation) matrix summarized the between-subject similarity of activation maps (Figure 1c). Next, a hierarchical cluster analysis was applied on all individual activation maps, which identified homogeneous subgroups of maps for each condition. Within each activation map subgroup, we estimated the average activation map across all subgroup members, or subtype map (Figure 1d).

Finally we computed the spatial similarity (correlation) of each individual activation map in the discovery sample with each of the identified activation subtypes (Figure 1e). The estimated spatial correlation coefficient was called the 'subtype weight' and each individual had a subtype weight for each subtype, ranging from -1 (perfect anticorrelation of the individual and the subtype map) to +1 (perfect correlation of the individual and subtype map). The subtype weights represent a continuous measure of similarity between a given individual activation map and a given subtype map. Unlike discrete assignments made by the hierarchical clustering, the weights are a soft assignment and can be seen as a dimension reduction of the individual maps, rather than pure categorical summary. It has been argued by several prior studies that such continuous summaries are more appropriate than discrete assignments (Badhwar, Peggy McFall, et al., 2019; Zhang et al., 2016), and we used the subtype weights as our primary metric for all subsequent statistical analyses (Figure 1f). 


\section{Clustering behavioral measures}

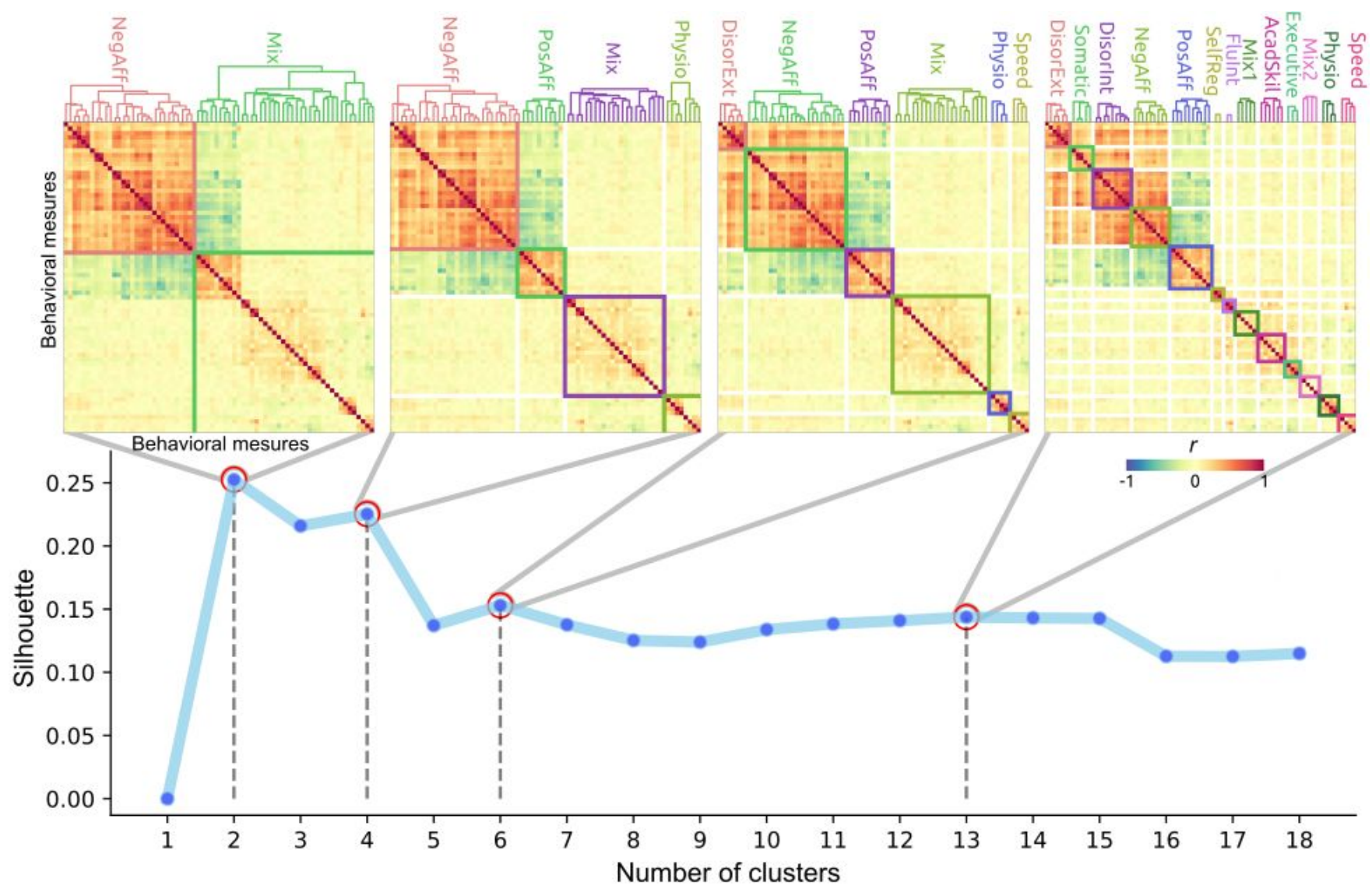

Figure 2 - Behavioral dimensionality reduction. A correlation matrix of a collection of 74 behavioral tests are clustered using k-means. The Silhouette plot shows 4 local maxima of cluster separation. The 13 clusters cut off are Speed: task reaction time measures, Physio: physiological measures, Mix2 and Mix1: a mixed categories of measures, Executive: working memory and cognitive flexibility measures, AcadSkil: language and vocabulary test along with academic completion measure, Flulnt: fluid intelligence measures, SelfReg: working memory and cognitive flexibility measures, PosAff: positive affect measures, NegAff: negative affect measures, Disorlnt: internalized disorders, Somatic: symptoms, problems or sensory manifestation related to the body, DisorExt: dysregulated externalized behaviour.

The majority of out-of-scanner HCP behavioral measures were composed of items from the NIH toolbox (NIH Toolbox, 2015). The behavioral measures were categorized into several domains that included measures of alertness, cognition, emotion, personality, motor and sensory processes in healthy individuals for a total of 74 items (Supplementary figure Sup2 gives details of individual behavioral measures). Since behavioral measures belonged to different categories, we reduced the number of items from 74 into 13 categories using a K-means clustering technique. Because participants were not statistically independent, but clustered into families, we first extracted the correlation between each behavioral measure using the Solar Eclipse polygenic model ${ }^{6}$. This model allowed us to control for the effect of family, which could otherwise bias the correlation between phenotypes. The number of categories was derived by a silhouette analysis, that characterized how 
well separated categories were for different numbers. The silhouette plot identified 4 local maxima of cluster separation, and we used the richest cluster solution, featuring 13 categories (see Figure 2). A first set of categories included behavioral assessments related to negative affect (NegAff), dysregulated externalized behavior (DisorExt), symptoms, problems or sensory manifestation related to the body (Somatic), and internalized disorder (Disorlnt). A second set of categories included physiological measures like blood pressure and body mass index (Physio), as well as reaction time measures (Speed). The third set of categories included items related to self regulation (SelfReg), working memory and cognitive flexibility (Executives), fluid intelligence group (Flulnt), language and vocabulary test along with academic completion measure (AcadSkil), and two mixed categories (Mix1, Mix2). The fourth and final category was composed of measures related to positive affect (PosAffect), which was identified robustly using 4, 6 or 13 clusters. Overall, the out-of-scanner behavioural assessments collected by HCP seemed to cluster into 13 easily interpretable categories, and we performed further analyses on the average scores within each category (after normalization of the scores for zero mean and unit variance across subjects).

\section{Phenotype definitions for heritability and pleiotropy estimates}

Brain phenotypes: Subtype weight measures were considered as a brain phenotype since they represented how spatially similar or dissimilar an individual activation map was to the subtype maps. These subtype weights were used as traits to estimate heritability, phenotypic correlation and genetic correlation. Each subject had 5 subtypes weights (Figure 1e) for each task condition (total of 105 brain phenotypic measures per subject).

Out-of-scanner behavioral phenotype: We used the 13 clustered out-of-scanner behavioral measures (Figure 2) to first estimate their heritability, and then to associate with brain phenotypes (subtypes weights) in terms of phenotypic correlation followed by genetic correlation (See supplementary material figure Sup2 for detailed individual behavioral measures).

In-scanner tasks performance phenotype: In-scanner tasks performance (15 measures) were also used to estimate heritability and to associate with brain phenotypes. For each task condition, we used the in-scanner accuracy measure and the reaction time, except the motor one which does not have accuracy measure. Supplementary Table 1 shows the complete list of in-scanner task performance measures used in this study. 


\section{Univariate analysis of additive genetic variance.}

For the heritability estimates, we used the Sequential Oligogenic Linkage Analysis Routines (SOLAR) Eclipse software package (http://www.nitrc.org/projects/se_linux). SOLAR uses maximum likelihood variance decomposition methods. The covariance matrix $\Omega$ for a pedigree is given by equation (1):

$$
\Omega=2 \cdot \Phi \cdot \sigma_{\mathrm{g}}^{2}+\mathrm{I} \cdot \sigma_{\mathrm{e}}^{2}
$$

where $\sigma_{g}^{2}$ is the genetic variance due to the additive genetic factors, $\phi$ is the kinship matrix representing the pairwise kinship coefficients among all individuals, $\sigma_{\mathrm{e}}^{2}$ is the variance due to individual - unique environmental effects and measurement error, and $I$ is an identity matrix (under the assumption that all environmental effects are uncorrelated among family members). Narrow sense heritability is given by equation (2) and is defined as the fraction of phenotypic variance $\sigma_{p}^{2}$ attributable to additive genetic factors:

$$
\mathrm{h}^{2}=\sigma_{\mathrm{g}}^{2} / \sigma_{\mathrm{p}}^{2}
$$

The variance parameters are estimated by comparing the observed phenotypic covariance matrix with the covariance matrix predicted by kinship (Almasy \& Blangero, 1998). Significance of the heritability estimate is tested by comparing the likelihood of the model in which $\sigma_{g}^{2}$ is constrained to zero with that of a model in which $\sigma_{g}^{2}$ is estimated. Twice the difference between the log-likelihoods of these models yields a test statistic, which is asymptotically distributed as a $1 / 2: 1 / 2$ mixture of $\chi^{2}$ variables with 1 degree-of-freedom and a point mass at zero. Prior to the heritability estimation, all phenotype (brain and behavioral phenotypes) were adjusted for covariates including sex, age and FD. Inverse Gaussian transformation was also applied to ensure normality of the distribution. Outputs from SOLAR included the heritability estimate $(\mathrm{H} 2 r)$, the significance value $(p)$, and the standard error for each trait (SE).

\section{Bivariate genetic analyses}

We used Equation (3) to simultaneously quantify the shared genetic variance and the phenotypic correlation between brain phenotypes (weights) and behavioral measures (in and out-of-scanner behavioral measures). To assess this relationship, we used SOLAR, relying on the following model:

$$
\rho_{p}=\sqrt{h_{a}^{2}} \sqrt{h_{b}^{2}} \cdot \rho_{g}+\sqrt{1-h_{a}^{2}} \sqrt{1-h_{b}^{2}} \cdot \rho_{e}
$$


where Pearson's phenotypic correlation $\rho_{\mathrm{p}}$ is decomposed into $\rho_{\mathrm{g}}$ and $\rho_{\mathrm{e}} . \rho_{\mathrm{g}}$ is the proportion of variability due to shared genetic effects and $\rho_{\mathrm{e}}$ that due to the environment, while $h_{a}^{2}$ and $h_{b}^{2}$ correspond to the previously defined narrow sense heritability for phenotypes $a$ and $b$, respectively. In our case, one corresponds to the heritability of subtypes weight while the second is the heritability of one of our behavioral scores.

\section{Results}

\section{Activation maps for each of the 7 tasks have good face validity are consistent with previous studies}

Activation group maps from each task condition showed the expected pattern of activation/deactivation related to each condition, and were visually similar to HCP maps from an introductory paper on tfMRI that were generated on a subset of the sample used here, and a different preprocessing strategy (Barch et al., 2013). Due to space limitations, we report here only activation maps for the Motor and Language tasks, (see Supplementary Material Figure Sup3 for all of the 7 tasks). In the Motor task, group functional activation patterns had cortical activation peaks located in the primary sensorimotor cortex, with a gradient of activation along the central sulcus that followed know principles of somatotopic organization: tongue in medio-dorsal position, hand in the area located next to the superior frontal sulcus, and foot in ventral position. Importantly, cortical activation peaks were located in the left hemisphere for right foot or hand movement, and in the right hemisphere, for left foot or hand movement, replicating the known decussation of motor cortico-spinal tract. These observations were all consistent with previous works (Grodd et al., 2001). The language group activation map (contrast of story condition against math condition) revealed a large fronto-temporo-parietal system, mostly left lateralized, with prominent activation in Brodman's areas 44/45 (Broca's area, associated with language production) and Brodman's area 22 (Wernicke's area, associated with language comprehension), as well as the medial temporal cortex and the temporal pole, in accordance with previous descriptions of the language network (Price, 2012; Friderici, 2016; Duffau, 2016). Overall, the group-level activation maps identified distributed brain networks with excellent biological plausibility, and were consistent with previous results from the literature, including those which directly examined HCP data using different analytical choices. 

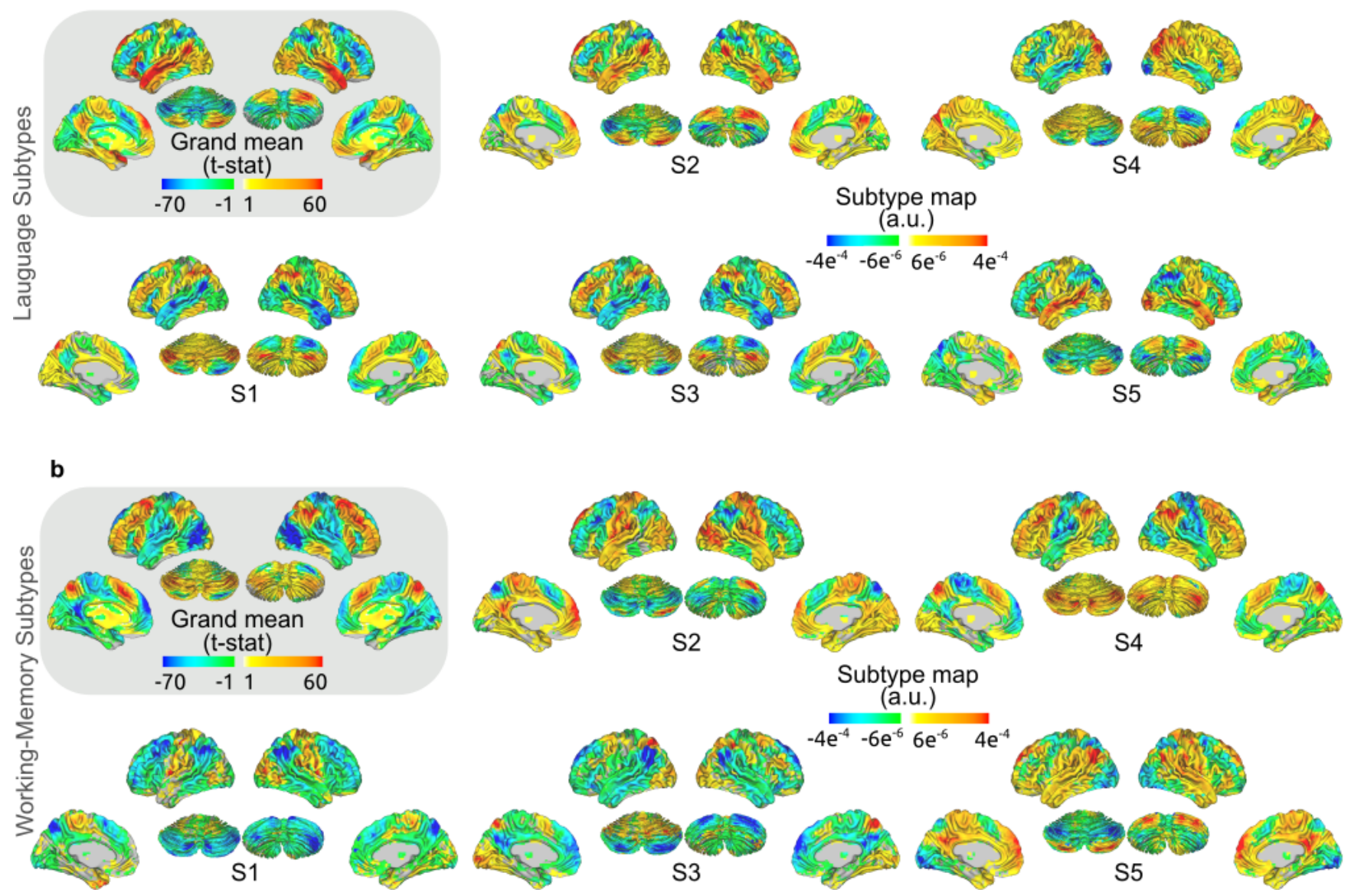

Figure 3 - Subtypes of activation maps - a: Language subtypes - The grand mean for the language statistical parametric maps revealed a cluster of high activation in the superior temporal gyrus during the "Story minus Math" condition. Subtype maps 1 to 5 (S1-5) were defined as the average map of each subgroup or cluster. Subtypes shows systematic deviation from the grand mean that range from a predominantly 'deactivating' pattern towards a more 'activating' pattern of brain activity b: Working memory subtypes - The grand mean for the working memory statistical parametric maps revealed a cluster of high activation in the shows bilateral activation in the superior frontal gyrus, middle frontal gyrus, inferior frontal gyrus and the inferior parietal lobule during the "0-Back minus 2-Back" condition. Subtype maps 1 to 5 (S1-5) were defined as the average map of each subgroup or cluster. They show activations and deactivations patterns along the superior frontal gyrus (BA 6), middle frontal gyrus (BA 9 and 10), inferior frontal gyrus (BA 47) and the inferior parietal lobule (BA 40).

\section{Individual activation maps cluster in subgroups with similar spatial}

\section{distribution}

We clustered the individual activation maps for each fMRI task condition separately in order to form subgroups of individuals that share similar spatial distribution within the same fMRI task condition. A subtype map was defined as the average activation map of each subgroup (Figure 1d). Figure 3 shows the 5 subtypes for the language and working memory tasks along with the group activation maps, which is the grand mean of all available subjects. For the language task (Figure 3a), the group map revealed a typical fronto-temporo-parietal system, but the subtype maps showed systematic deviations from this pattern. Subtype $\mathrm{S} 1$ exhibited stronger activation of fronto-parietal cortices, and underactivation of the bilateral temporal cortices, which mirrored the grand mean. Subtype S2 showed 
hyper activation of the temporo-parietal junction, along with smaller clusters along the bilateral superior temporal gyri and inferior frontal gyri. Subtype S3 closely resembled S1. Subtype S4 showed a high activation cluster in the superior occipital and superior parietal lobes, while deactivating the main regions of the grand mean. Subtype S5 showed similarity to the grand mean, and was thus composed of subjects who shared a prototypical spatial distribution of activation, yet more strongly activated these regions than other subjects.

For the working memory task (Figure 3b), subtypes showed bilateral activation/deactivation in the superior frontal gyrus (BA 6), middle frontal gyrus (BA 9 and 10), inferior frontal gyrus (BA 47) and the inferior parietal lobule (BA 40). The difference observed within subtypes from the same task are highlighted by the difference of activation or deactivation within these brain regions. For example, Subtypes S1 and S2 are mirrored versions of the grand mean activation map of the working memory task, while Subtypes S3 and S4 present the same pattern of activation as the grand mean with slight difference in the intensity. Overall, group activation maps appeared to be an aggregation of heterogeneous activation subgroups. This conclusion generalized to all tasks, and is illustrated in more detail in the supplementary material figure Sup4 for the remaining 5 tasks.

a

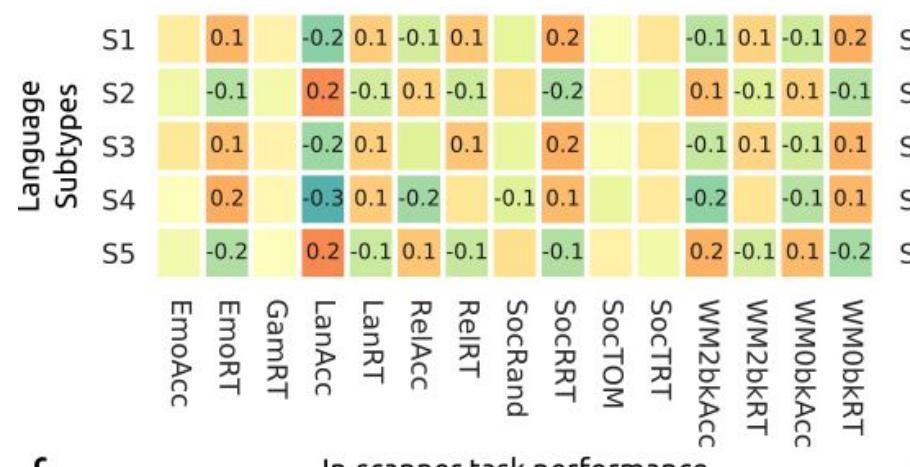

c In-scanner task performance b

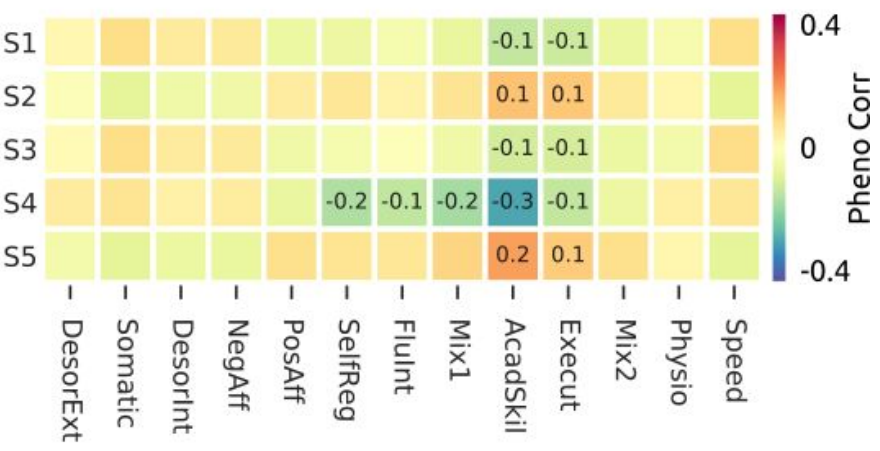

d

Out of scanner behavioral clusters

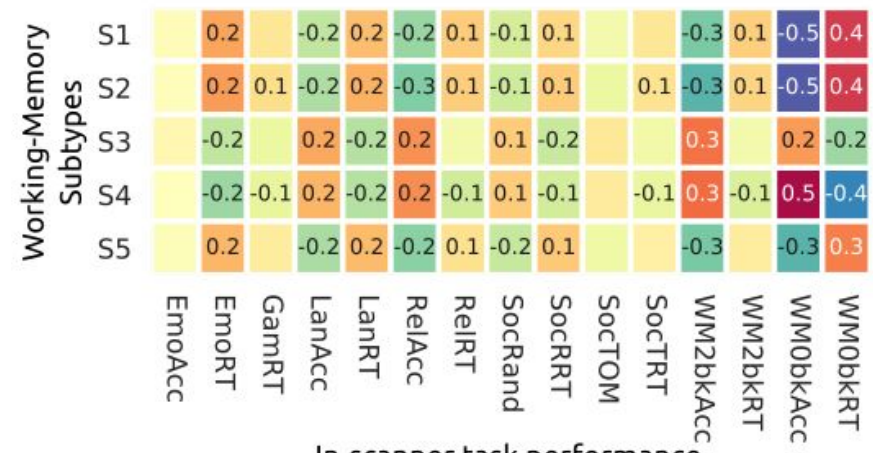

In-scanner task performance

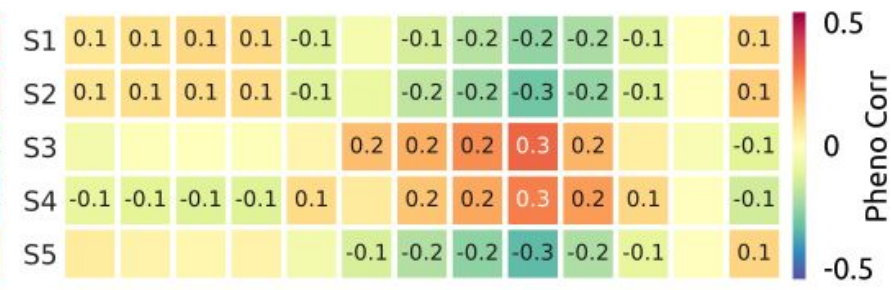

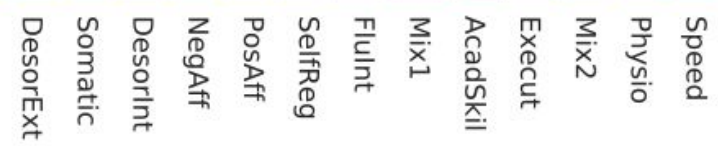

Out of scanner behavioral clusters

Figure 4 - Phenotypic correlation between brain subtypes and behavioral measures - a: Correlation between language subtypes and in-scanner task performance. The highest correlation estimates are between the language task accuracy (LanAcc) measure and subtypes weights (S1-S5). See supplementary material Table 1 for a list of abbreviations used for the in-scanner task performance. $\mathbf{b}$ - Correlation between language subtypes and out-of-scanner behavioral measures: The highest correlation estimates are between the academic skills (AcadSkil) behavioral cluster and subtypes weights. See supplementary material figure Sup2 for a list of abbreviations used for the behavioral clusters. $\mathbf{c}$ - Correlation between working memory subtypes and in-scanner task performance. The highest correlation estimates are between the working memory task 
accuracy measures and all subtypes weights. d - Correlation between working memory subtypes and out-of-scanner behavioral measures. The highest correlation estimates are between the academic skills (AcadSkil) behavioral cluster and all subtypes weights.

\section{Language and working-memory subtypes weights associate with behavioral phenotypes}

We associated subtypes weights from the 7 tasks with behavioral measures, collected both inside and outside the scanner. Only the weights of the language and the working memory task subtypes showed significant correlations with behavioral phenotypes (See figures Sup5 and Sup6 in supplementary material). Notably, subtype weights of both tasks associated most strongly with performance during these tasks inside the scanner. For example, the language subtype weights showed the highest association with language accuracy performance (figure 4a), and the working memory subtype weights associated most strongly with working memory related task performance (figure 4c). Subtypes weights were also associated with meaningful outside-of-the-scanner behavioural measures. For example, subtype weights of the language task associated most strongly with the cluster of academic skills (figure 4b), and working memory subtype weights associated most strongly with clusters of fluid intelligence, Mix1, academic skills and executive measures (figure 4d). None of the remaining tasks subtype weights showed significant correlation with their corresponding in-scanner task performance (see supplementary material figures Sup5 and Sup6). 

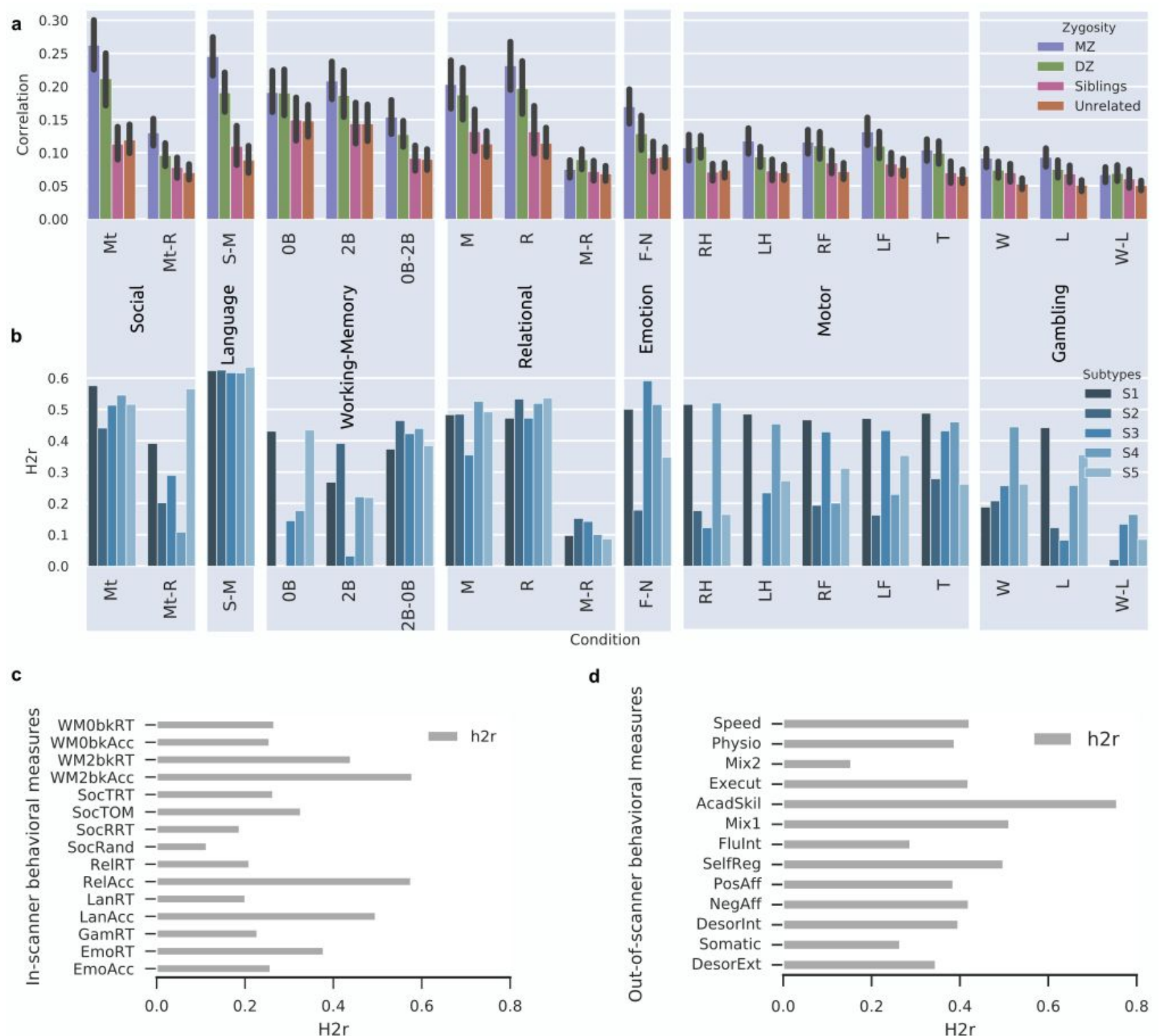

d

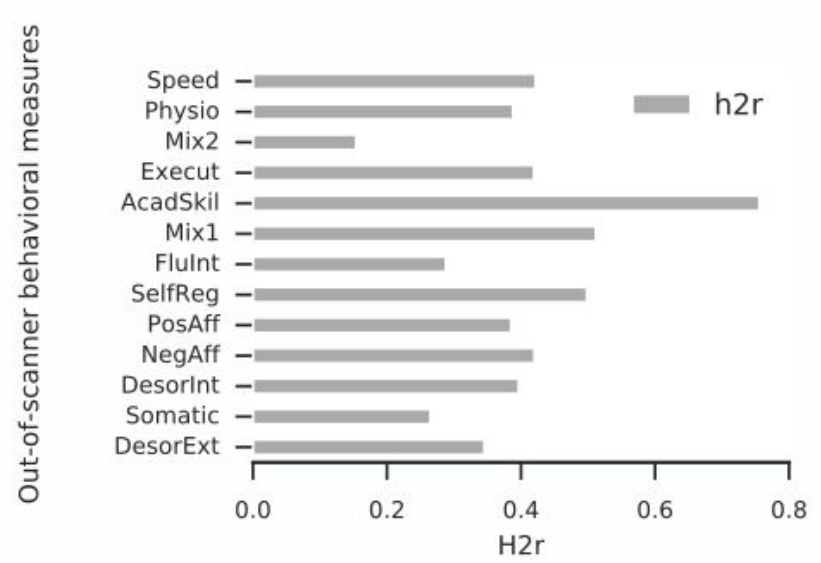

Figure 5 - Heritability estimate - a: Similarity between activation maps by relationship - Average correlation of brain activation maps between pairs of MZ twins, DZ twins, siblings and unrelated individuals. The magnitude of correlations varied substantially across task domains, but a dosage effect was observed in almost every task, with correlation for $\mathrm{MZ}$ twins greater than $\mathrm{DZ}$ twins, greater than siblings or unrelated individuals. b: Heritability of subtype weights for all HCP tasks. Significant heritability was identified for the 7 HCP tasks: gambling Task (W: Win, L: Loss, W-L: Win vs Loss), language task (S-M: Story vs Math), motor task (LH: Left Hand, LF: Left Foot, T: Tong, RF: Right Foot, LF: Left Foot), social task (Mt: Mental, Mt-R: Mental vs Random), working memory task (2B: 2-Back, OB: 0-Back, 2B-0B: 2-Back vs 0-Back). Relational task (M: Match, R: Relational, M-R: Match vs Relational) and the emotional task (F-N: Fear vs Neutral). Each task condition was represented by 5 subtypes' weights. Heritability estimate $(\mathrm{H} 2 \mathrm{r})$ on these subtype weights ranged from 0 to 0.62 . c: Heritability of in-scanner task performance. Heritability ranged from 0.12 to 0.57 with the highest estimates for working memory and relational accuracy scores. See supplementary material Table 1 for a detailed description of in-scanner behavioral labels d: Heritability of out-of-scanner behavioral clusters. Heritability ranged from 0.15 to 0.75 with the highest estimate for academic skills (AcadSkil). See supplementary material figure Sup2 for a list of abbreviations used for the clustered behavioral measures. 


\section{Heritability of subtypes weights ranges from weak to strong}

We then estimated the heritability of brain subtypes weights. We first quantified the intersubject spatial similarity (correlation) between individual activation maps for all MZ twins, DZ twins, siblings pairs and unrelated pairs of subjects, separately. The similarity measures ranged from $r=0.05-0.25$, with a marked variability between tasks. The tasks with the highest inter-subject similarity (social, language, working memory, relational, emotion) showed a clear dosage effect of shared genetics (MZ > DZ > siblings and unrelated, see figure 5a), suggesting genetic control over activation maps. We then tested the heritability of subtypes weights. Significant estimates of additive genetic components were found on subtype weights for all 7 tasks. The heritability strength varied widely across tasks, from close to $\mathrm{h}^{2}=0$ - 0.62 (see figure $5 \mathrm{~b}$ ). Some tasks had all associated subtypes highly heritable, e.g. the language task presented the strongest heritability estimates, above $h^{2}=0.6$ for all 5 subtypes, followed by relational, working memory and social tasks. As expected, these activation tasks were also the ones with the highest inter-subject spatial similarity of activation maps and the clearest dosage effects. The gambling task presented the lowest estimate of heritability among all 7 tasks, particularly on the win versus loss contrast $\left(h^{2}=0-0.18\right.$, Figure $\left.5 b\right)$. The same task also presented the lowest intersubject correlation ( $r$ $=0.05-0.08$, Figure 5a). Overall, some subtypes weights were found to be highly heritable, and the level of heritability was markedly heterogeneous across tasks and across subtypes.

\section{In-scanner and Out-of-scanner behavioural measures are heritable}

Before testing for shared genetic control over brain activation and behaviour, we tested for genetic control over behavioral measures alone. Both in-scanner and out-of-scanner behavioral measures showed significant estimates of additive genetic effects. Regarding the in-scanner performance measures, the highest heritability measures were for the relational task $\left(h^{2}{ }_{\text {RelAcc }}=0.57\right)$ and working memory task $\left(h_{\text {WM2bkAcc }}^{2}=0.57\right)$, while the heritability of the remaining measures ranged from $h^{2}=0.12-$ 0.5 (lowest SocRand), see Figure 5c. Among the out-of-scanner behavioural skills, the Academic skills subgroup had the highest heritability estimate $\left(\mathrm{h}^{2}{ }_{\text {AcadSkil }}=0.77\right)$, followed by Mix1 $\left(\mathrm{h}^{2}{ }_{\text {Mix } 1}=0.52\right)$ and SelfReg $\left(h_{\text {SelfReg }}^{2}=0.50\right)$. The remaining subgroups had heritability estimates ranging from $h^{2}=0.12-$ 0.40 (lowest Mix2), see Figure $5 \mathrm{~d}$. 
a

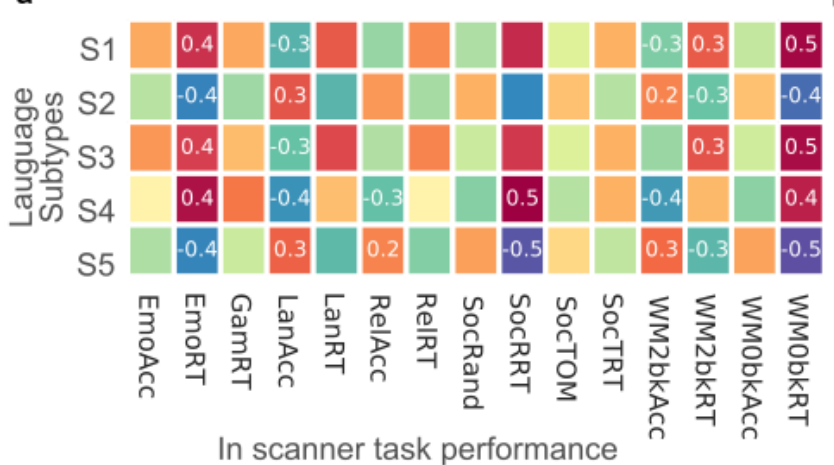

In scanner task performance

c
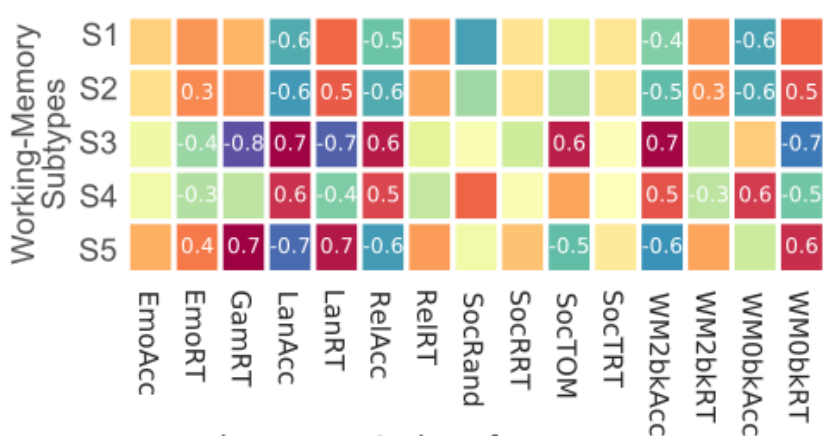

In scanner task performance b

\section{S1 \\ S2 \\ S3 \\ S4 \\ S5}

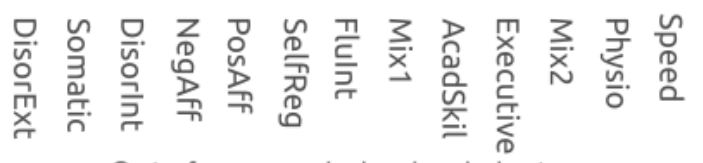

Out of scanner behavioral clusters

d

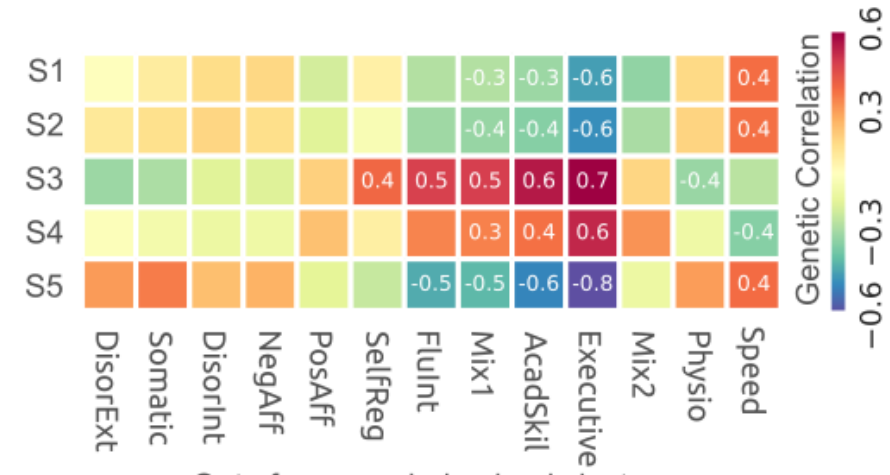

Out of scanner behavioral clusters

Figure 6 - Genetic correlation between brain phenotypes and behavioral phenotypes. a - Genetic correlation between language subtypes and in-scanner behavioral tests: The highest genetic associations are between the working memory reaction time and the five language subtypes weights. See supplementary material Table 1 for a list of abbreviations used for the in-scanner task performance. $\mathbf{b}$ - Genetic correlation between language subtypes and out-of-scanner behavioral measures. All five language subtypes weights are genetically correlated with Executive function behavioral cluster, but the highest genetic correlation is between Mix2 and subtypes S1,S3 and S5 $\left(\rho_{g S 1}=-0.5, \rho_{g S 3}=-0.5\right.$ and $\left.\rho_{g S 5}=0.5\right)$. See supplementary material figure Sup2 for a list of abbreviations used for the behavioral tests. $\mathbf{c}$ - Genetic correlation between working memory subtypes and in-scanner behavioral tests. Working memory subtypes are genetically correlated with all tasks either with reaction time or accuracy measures. Genetic correlation range from -0.8 to 0.7 . d - Genetic correlation between working memory subtypes and out-of-scanner behavioral measures: Genetic correlation estimate between working memory subtypes weights and out-of-scanner task performance shows the highest value for Executive function behavioral cluster followed by academic skills.

\section{Subtypes weights are genetically associated with behaviour}

We performed a bivariate genetic analysis to quantify the shared genetic influence (genetic correlation) between subtypes weights for each of the 7 tasks and the behavioral measures inside and outside the scanner. We found significant genetic correlations between the subtypes weights and behavioral measures, especially for the language and working memory tasks (Figure 6), that we report in this section. Other genetic association tests are reported in Supplementary Material Sup5 and Sup6.

Figure 6-a summarizes the genetic correlation between the language brain activation subtype weights and in scanner task performance. The five language subtypes showed significant shared genetic variance with in-scanner language performance (LanAcc, $\rho_{g}=-0.4-0.3$ ), Emotional task reaction time (EmoRT, $\left.\rho_{g}=-0.4-0.4\right)$ and working memory reaction time (WMObkRT, $\left.\rho_{g}=-0.5-0.5\right)$. 
Figure 6-b shows the genetic correlation between the language subtype weights and out-of-scanner behavioral clusters. The behavioural measures of executive functions were genetically correlated with all 5 subtype weights $\left(\rho_{g}=-0.3-0.4\right)$, and the AcadSkil behavioural measures were genetically correlated with weights of 4 of subtypes (AcadSkil, $\rho_{g}=-0.4-0.4$ ). Mix2 (Sensory-motor and emotion) showed the highest genetic correlations with subtypes S1, S3 and S5 ( $\rho_{g S 1}=-0.5, \rho_{g S 3}=-0.5$ and $\rho_{g S 5}$ $=0.5)$, yet this behavioural subtype was not highly heritable $\left(h^{2}=0.12\right)$. Finally, subtype weight $S 4$ of the language task (figure 6-b) shared genetic influences with 6 of the behavioral clusters (SelfReg, Flulnt, Mix1, AcadSkil, Executive and Physio).

The five working memory subtypes weights (figure 6-c) also showed significant shared genetic variance with in-scanner language (LangAcc, $\rho_{g}=-0.7-0.7$ ), relational (RelAcc, $\rho_{g}=-0.6-0.6$ ) and working memory 2back (WM2bkAcc, $\left.\rho_{g}=-0.6-0.7\right)$ tasks performances. Figure 6d shows genetic correlation between the working memory subtype weights and clusters of out-of-scanner behavioral measures. Executive, AcadSkil and Mix1 behavioral clusters were genetically correlated with all 5 WM subtype weights (-0.8 to 0.7 ). The remaining tasks did not present any significant associations between subtype weights and other behavioral measures inside and outside the scanner.

In summary, we found moderate to strong genetic associations between brain subtypes weights and behavioural measures (in-scanner and out-of-scanner). These associations were particularly strong only for the language and working memory subtypes weights. Subtypes weights from the remaining tasks did not reach significant associations with behavioral measures inside and outside the scanner.

\section{Discussion}

The goal of this work was to identify subtypes of brain activation and test their phenotypic and genetic association with behavioral measures. Our results demonstrated that subtypes were moderately to highly heritable. Only the language and working memory subtypes associated with behavioral measures, phenotypically and genetically. Our findings emphasize the importance of considering both inter-individual differences and commonalities for the understanding of task-related brain activation, and call into question the validity of using group averages of brain activation patterns.

\section{Brain subtypes are associated with behavioral measures}

Unsupervised clustering methods are a practical means of characterizing heterogeneous data by delineating homogeneous subpopulations. Subtyping has been predominantly applied to investigate brain subtypes linked to brain disorders but little is known about brain subtypes in a healthy population. Our results on subtypes of brain activation in a healthy sample show that we could distinguish subtypes of similar brain activation with good face validity. Moreover, the weights of these subtypes were shown 
to be correlated with some behavioral measures. Our results on brain activation subtype are hardly comparable with studies that used group activation maps, but some consistency could be observed in terms of the core regions activated in both studies. For example in the comprehension language task, our results replicated involvement of bilateral temporal areas in speech comprehension, which has been shown in prior studies (Hickok \& Poeppel, 2007). As expected, the superior temporal gyrus/sulcus and medial temporal gyrus were involved in narrative comprehension symmetrically in both hemispheres (AbdulSabur et al., 2014) in the group activation map (see figure 3-a grand mean). The 5 subtypes of the language task also presented resemblance with the group average map with marked differences in the combination of regions activated or deactivated that is specific to each subtype. For example in figure 3a, S1 and S5 are mirrored versions of one another and show opposing association with in scanner language task performance measures (figure $4 a$, Correlation for $S 1=-0.2$ and S5=0.2).

In this study, only the language and working memory subtypes were associated with a set of behavioral measures. The language subtypes showed the highest correlation with in-scanner performance accuracy for the language task (correlation range from -0.25 to 0.3 ). Le Guen and colleagues found the same associations between the median activation of the contrast STORY-MATH maps from HCP language task and the behavioral measure inside the scanner (RoP, range from -0.23 to 2.28). They also found correlation with behavioral measures like fluid intelligence, working memory, vocabulary comprehension and reading decoding (correlation range from -0.25 to 0.25 ). Their results are consistent with our finding about the correlation on subtypes of language and clustered behavioral measures (figure $3 b$ ). Figure 3b shows that language subtypes are associated mostly with AcadSkil cluster (correlation range from -0.3 to 0.2 ) but less with fluid intelligence cluster (correlation $=0.1$ ). Since our behavioral measures are clustered, it is hard to make a direct comparison with other studies that used HCP behavioral measures to associate with brain activation. Nevertheless, we still find some concordance in the association between some behavioral phenotype and brain phenotypes for language and working memory tasks. For the working memory task, group activation maps (figure 3b, Grand mean) showed typical activation in bilateral dorsal and ventral prefrontal cortex, dorsal parietal cortex and dorsal anterior cingulate. Further, we also see deactivation in the default mode network, including medial prefrontal cortex, posterior cingulate, and the occipital-parietal junction, same as shown in the HCP Consortium paper for task activation (Barch et al., 2013). The working memory subtypes also resemble the grand mean but with notable differences in the combination of activation and deactivations for the above mentioned regions. As we mentioned above for language subtypes, it is hard to compare our working memory subtypes to other studies since they used group activation maps. Nevertheless, our results for working memory subtypes are consistent with Miller and colleagues (2009) who used memory retrieval tasks on 14 subjects and looked at intra and inter-individual variability in brain activation patterns. They found that activity patterns of the same individual performing different tasks were more similar than activity patterns of different individuals performing the 
same task. They also found that individual differences in decision criteria on a recognition test predicted the degree of similarity between any two individuals' patterns of brain activity.

Our working memory subtypes also showed phenotypic association with behavioral measures. In-scanner task performance showed the highest associated with working memory reaction time and accuracy (figure 4c), which is consistent with results from Saliasi (2014) and colleague who found higher BOLD response in the VLPFC was associated with increased performance accuracy in older adults, in both the baseline and the more complex task condition. Literature on association with working memory brain activation and behavior, mostly found age related difference (Yaple et al., 2019). In our study, we cannot test significant age differences since age in the HCP sample ranges from 22 to 35 , which is considered a young adult. But we did find associations with behavioral measures outside the scanner. These associations were mainly composed of measures related to cognitive functioning (fluid intelligence, academic skills and executive functions). To our knowledge this is the first study showing association between brain activation from the n-back memory task and behavioral measures outside the scanner. The remaining 5 tasks activation subtypes (motor, relational, gambling, emotional and social) did not show any association neither with task performance nor with any behavioral measures.

\section{Heritability of brain subtypes and behavioral measure}

Our results showed that most subtypes of brain activation from the 7 tasks had moderate to high genetic influence. The language task showed the highest heritability estimate (mean $\left.\mathrm{h}^{2}=0.52\right)$ which is in line with previously reported non-imaging studies on heritability of the language ability (Stromswold, 2001). A meta-analysis of the available evidence concerning a genetic basis for language (Stromswold, 2001) concluded that genetic factors continue to be a predictive element of language ability into adulthood. Brain imaging heritability of language studies likewise indicates a large heritability. We found that superior temporal gyrus and sulcus, inferior frontal gyrus, supplementary motor area, medial superior frontal gyrus, precuneus and middle frontal gyrus were overall highly activated in the 5 subtypes of the language task and these subtypes were heritable. This is in line with previous findings on the heritability of the HCP language task activation maps. Le Guen and colleagues (2018), using the 900 release of $\mathrm{HCP}$, found heritability of language brain activation to range from 0.22 to 0.55 in brain areas overlapping with our study. Abbas Babajani-Feremi ((2017) also found high heritability for HCP language tasks ( $\mathrm{h} 2=0.36$ ). Although our own heritability estimates are higher $\left(\mathrm{h}^{2}=0.52\right)$, we should note that there is a difference on the nature of brain phenotype used (subtypes weights vs voxelwise heritability).

For working memory brain activation subtypes, heritability (0.38 to 0.46$)$ is in line with previous studies showing that a significant proportion of the variance in working memory activation maps may be attributed to genes (Blokland et al., 2011, 2017). These studies from Blokland and colleagues used 
voxelwise heritability estimate on region of interest and showed that there is a significant and substantial genetic influence on working memory task-related activation across the brain, with genes accounting for up to $65 \%$ of the variance, averaging $33 \%$. For the social, emotional, gambling, motor and relational tasks, we are not aware of any $\mathrm{fMRI}$ studies that estimated heritability of activation maps using these same tasks as HCP one. Nevertheless, in the social and gambling domains, some studies explored heritability of task activation fMRI. Van der Meulen and colleagues (2018) showed that children experiencing self-inclusion was associated with activity in anterior cingulate cortex, insula and striatum, but this was not significantly explained by genetics or shared environment. Our results for the social task (mental minus random contrast) showed low to high heritability estimates (0.1 to 0.55 ), depending on the subtype. This difference between our study and Van Der Meulen results could be attributed to the nature of social tasks used. Our study used a social task designed to capture the neural correlates of theory of mind, while the other study was interested in social inclusion/exclusion aspects. For the gambling task, Rao and colleagues (2018) investigated the heritability of risk taking and the genetic influence on individual variation in risk-related brain activation. They used a balloon analogue risk task to assess individual risk-taking behavior in a sample of 244 pairs of young adult twins. They found that there was a moderate heritability $(41 \%)$ of risk taking. Their results were consistent with only two subtypes (S4 win condition and S1 loose condition, figure 3a), the other subtypes had lower heritability estimates. This difference between their results and our results could be, first, attributed to the difference in tasks used and second, our gambling task showed the lowest intersubject correlation between individual brain maps compared to the other tasks. This lack of correlation may be interpreted as the gambling task used in HCP is less engaging than the other tasks. This phenomenon is largely revealed by the field of neuro-cinematic, where studies use movies as a stimulus for the tfMRI, see (Hasson et al., 2008).

Our results on heritability of behavioral measures are also consistent with the literature. For the out-of-scanner behavioral measures, a previous study (Han \& Adolphs, 2019) identified nine latent factors among the HCP behavioral measures (positive social ability, negative affect, general intelligence, self-regulation, attention and processing speed, agreeableness, self-efficacy, language and communication, and competitiveness). The estimated heritability for these nine latent factors ranged from 0.1 to 0.6 , using Falconer's formula. Their results are close to our findings, since we also reduced dimensionality of our data and found 13 latent variables (out of 74 measures) and found heritability that ranged from 0.2 to 0.8 . In-scanner task performance heritability estimates were also consistent with studies that used the same HCP data (Babajani-Feremi, 2017; Le Guen et al., 2018).

\section{Genetic correlation between brain subtypes and behavioral phenotypes}

The last goal of this work was to estimate the shared genetic influence between brain phenotypes and behavioral phenotypes. Our results showed significant genetic correlation between brain phenotypes 
and behavioral phenotypes. Le Guen and colleagues (2018) used HCP data to perform bivariate genetic analyses between the neural activations of the language task and behavioral scores, corresponding to the fMRI task accuracies, fluid intelligence, working memory and language performance. They observed that several parts of the language network along the superior temporal sulcus, as well as the angular gyrus belonging to the math processing network, are significantly genetically correlated with these indicators of cognitive performance. Their findings are consistent with our result of genetic correlation between brain activation and behavioral measures. We both found significant association between language brain phenotypes and in scanner language task performance accuracy, and with cognitive abilities. In addition we found that some of our language subtypes were also associated with task performances and reaction time from other non language tasks, namely the working memory, emotional social and the relational tasks. The same genetic association also happened between working memory brain phenotypes and behavioral ones. The observed genetic correlation between brain and behavioral phenotypes in this work shed light on the genetic root of individual variability. Subtypes are shown to have shared genetic aetiologie with behavioral measures. Our results are a step forward to understand the nature of inter-individual brain variability in relation to behavioral manifestation and their genetic link.

\section{Cognitive factors underlying brain subtypes?}

There are many possible factors that could be contributing to individual heterogeneity in brain activity, including individual differences in cognitive processing, psychological states or traits, physiology, anatomy, personality, and genetics. Kirchhoff and colleagues (2006) tested the neural correlates of different memory encoding strategies and found that self-reported use of strategies did capture significant variation in memory performance and regional brain activity. This suggests that different cognitive strategies used by the individuals to execute a given task may be reflected by homogeneous subgroups of activation patterns. Our finding on the correlation between subtypes and behavioral measures supports that hypothesis, in which brain phenotype (only for the language and working memory task) associated with the task performance of the related task. For example, in the language task (figure 4a), subjects who exhibited spatial brain activations pattern similar to the subtype activation map S1 have positively correlated behavioral scores of the in-scanner language task, while subjects who exhibited brain activation patterns similar to subtype 2 (S2), have negatively correlated behavioral scores for the in-scanner behavioral language task. We can speculate that subjects associated with subtype 1 have used different cognitive strategies to perform the language task compared to subjects associated with subtype 2. This strategy shifting and differences in normal population could be extended to brain disease studies. For example, is it possible that certain subtypes are more at risk for a specific brain disorder and are indeed using a less efficient cognitive strategy to get the task done. 
More direct characterization of cognitive strategies employed during tasks will be needed to shed more light on the cognitive and physiological basis of interindividual heterogeneity in brain activation.

\section{Limitations}

This study has some limitations. First, in this work we made the choice of preprocessing the HCP data and not using the HCP preprocessed data mainly to implement motion censoring. Since head motion is highly heritable, we opted for using the scrubbing method to ensure that time points with excessive motion are removed. Figure Sup1 in the supplementary material shows the decreased heritability estimate of head motion after applying scrubbing. Despite our efforts to reduce the effects of head motion, we still need to be careful in the interpretation of our result, since some effects of head motion may remain in the data and bias heritability estimate (Bolton et al., 2020).

Second, in this work, we could not accurately estimate the effect of the shared environment between siblings, since HCP did not provide the household information necessary to model it. We opted for using the mother ID as a household ID. This is not ideal, since split families (ex: divorced) are still considered to share the same familial environments, which is not necessarily the case. Our heritability estimates could be slightly over-estimated for that reason.

Third, we should caution about the possibly limited generalisability of our study since the majority of participants were Caucasian (European origin). HCP did offer information about the ethnicity of the participants but this information was not sufficient to use as confounding elements in our analysis.

\section{Future directions}

The recent efforts in associating subpopulation of brain disorders and brain patterns has led to a shift in the way we conceive a disease. These studies helped clarify the marked heterogeneity that exists in psychiatric illnesses by associating structural and functional subtypes with different clinical diagnostics for the same disorder. Studies showed significant associations between brain subtypes from structural measures and clinical diagnosis, but they lacked the explanation behind these associations. Our work on healthy populations could explain better the nature of those subgroups in the normal population by pointing to the biological underpinning of their manifestation, and their relationship with behavior. As a future direction, one could compare the distribution of the subtypes of activation maps with gene expression in the Allen brain atlas (Sunkin et al., 2013). Recent work by Grasby (2020) and colleagues explored a similar relationship by associating between cortical thickness and neurological, psychological, and behavioral traits. The highly polygenic architecture of the cortex suggests that distinct genes are involved in the development of specific cortical areas. Moreover, they found evidence that brain structure is a key phenotype along the causal pathway that leads from genetic variation to differences in general cognitive function. Similar work could be performed by focusing on the functional organisation of brain maps from subtypes. The results will help make sense of the relation between 
subtypes of brain activation, genetic and behavioral measure in our work. This will guide other studies on brain disorder, and what variables are worth looking for to explain subgroups of the same disease and their relation to behavioural phenotypes.

\section{Acknowledgements}

YB, AB, and PB are currently supported by the Canadian Consortium on Neurodegeneration in Aging (CCNA) and the Courtois Foundation. $A B$ is currently supported by a CIHR Postdoctoral Fellowship (funding reference number \#152548). At the start of the project $A B$ was supported by the Alzheimer Society of Canada Postdoctoral Fellowship. PB is a Research Scholar from the Fonds de Recherche du Québec, UV is supported by Estonian Research Council's MOBTP94 grant.

\section{References}

AbdulSabur, N. Y., Xu, Y., Liu, S., Chow, H. M., Baxter, M., Carson, J., \& Braun, A. R. (2014). Neural correlates and network connectivity underlying narrative production and comprehension: a combined fMRI and PET study. Cortex; a Journal Devoted to the Study of the Nervous System and Behavior, 57, 107-127.

Ad-Dab’bagh, Y., Lyttelton, O., Muehlboeck, J. S., Lepage, C., Einarson, D., Mok, K., Ivanov, O., Vincent, R. D., Lerch, J., Fombonne, E., \& Others. (2006). The CIVET image-processing environment: a fully automated comprehensive pipeline for anatomical neuroimaging research. Proceedings of the 12th Annual Meeting of the Organization for Human Brain Mapping, 2266.

Almasy, L., \& Blangero, J. (1998). Multipoint quantitative-trait linkage analysis in general pedigrees. American Journal of Human Genetics, 62(5), 1198-1211.

Babajani-Feremi, A. (2017). Neural Mechanism Underling Comprehension of Narrative Speech and Its Heritability: Study in a Large Population. Brain Topography, 30(5), 592-609.

Badhwar, A., McFall, G. P., Sapkota, S., Black, S. E., Chertkow, H., Duchesne, S., Masellis, M., Li, L., Dixon, R. A., \& Bellec, P. (2019). A multiomics approach to heterogeneity in Alzheimer's disease: focused review and roadmap. Brain: A Journal of Neurology. https://doi.org/10.1093/brain/awz384 Badhwar, A., Peggy McFall, G., Sapkota, S., Black, S. E., Chertkow, H., Duchesne, S., Masellis, M., Li, 
L., Dixon, R., \& Bellec, P. (2019). A Multiomics Approach to Heterogeneity in Alzheimer's Disease: Focused Review and Roadmap. medRxiv, 19008615.

Barch, D. M., Burgess, G. C., Harms, M. P., Petersen, S. E., Schlaggar, B. L., Corbetta, M., Glasser, M. F., Curtiss, S., Dixit, S., Feldt, C., Nolan, D., Bryant, E., Hartley, T., Footer, O., Bjork, J. M., Poldrack, R., Smith, S., Johansen-Berg, H., Snyder, A. Z., ... WU-Minn HCP Consortium. (2013). Function in the human connectome: task-fMRI and individual differences in behavior. Neurolmage, 80, 169-189.

Bellec, P., Lavoie-Courchesne, S., Dickinson, P., Lerch, J. P., Zijdenbos, A. P., \& Evans, A. C. (2012). The pipeline system for Octave and Matlab (PSOM): a lightweight scripting framework and execution engine for scientific workflows. Frontiers in Neuroinformatics, 6. https://doi.org/10.3389/fninf.2012.00007

Benhajali, Y., Badhwar, A., Spiers, H., Urchs, S., Armoza, J., Ong, T., Pérusse, D., \& Bellec, P. (2020). A Standardized Protocol for Efficient and Reliable Quality Control of Brain Registration in Functional MRI Studies. Frontiers in Neuroinformatics, 14, 7.

Blokland, G. A. M., de Zubicaray, G. I., McMahon, K. L., \& Wright, M. J. (2012). Genetic and environmental influences on neuroimaging phenotypes: a meta-analytical perspective on twin imaging studies. Twin Research and Human Genetics: The Official Journal of the International Society for Twin Studies, 15(3), 351-371.

Blokland, G. A. M., McMahon, K. L., Hoffman, J., Zhu, G., Meredith, M., Martin, N. G., Thompson, P. M., de Zubicaray, G. I., \& Wright, M. J. (2008). Quantifying the heritability of task-related brain activation and performance during the N-back working memory task: a twin fMRI study. Biological Psychology, 79(1), 70-79.

Blokland, G. A. M., McMahon, K. L., Thompson, P. M., Martin, N. G., de Zubicaray, G. I., \& Wright, M. J. (2011). Heritability of working memory brain activation. The Journal of Neuroscience: The Official Journal of the Society for Neuroscience, 31(30), 10882-10890.

Blokland, G. A. M., Wallace, A. K., Hansell, N. K., Thompson, P. M., Hickie, I. B., Montgomery, G. W., Martin, N. G., McMahon, K. L., de Zubicaray, G. I., \& Wright, M. J. (2017). Genome-wide association study of working memory brain activation. International Journal of Psychophysiology: 
Official Journal of the International Organization of Psychophysiology, 115, 98-111.

Bolton, T. A. W., Kebets, V., Glerean, E., Zöller, D., Li, J., Yeo, B. T. T., Caballero-Gaudes, C., \& Van De Ville, D. (2020). Agito ergo sum: Correlates of spatio-temporal motion characteristics during fMRI. Neurolmage, 209, 116433.

Brain Match. (2015). Zooniverse. https://www.zooniverse.org/projects/simexp/brain-match Caballero-Gaudes, C., \& Reynolds, R. C. (2017). Methods for cleaning the BOLD fMRI signal. Neurolmage, 154, 128-149.

Chen, B., Zhu, Z., Wang, Y., Ding, X., Guo, X., He, M., Fang, W., Zhou, Q., Zhou, S., Lei, H., Huang, A., Chen, T., Ni, D., Gu, Y., Liu, J., \& Rao, Y. (2018). Nature vs. nurture in human sociality: multi-level genomic analyses of social conformity. Journal of Human Genetics, 63(5), 605-619.

Collins, D. L., \& Evans, A. C. (1997). Animal: Validation and Applications of Nonlinear Registration-Based Segmentation. International Journal of Pattern Recognition and Artificial Intelligence, 11(08), 1271-1294.

Couvy-Duchesne, B., Blokland, G. A. M., Hickie, I. B., Thompson, P. M., Martin, N. G., de Zubicaray, G. I., McMahon, K. L., \& Wright, M. J. (2014). Heritability of head motion during resting state functional MRI in 462 healthy twins. Neurolmage, 102P2, 424-434.

Couvy-Duchesne, B., Ebejer, J. L., Gillespie, N. A., Duffy, D. L., Hickie, I. B., Thompson, P. M., Martin, N. G., de Zubicaray, G. I., McMahon, K. L., Medland, S. E., \& Wright, M. J. (2016). Head Motion and Inattention/Hyperactivity Share Common Genetic Influences: Implications for fMRI Studies of ADHD. PloS One, 11(1), e0146271.

Deipolyi, A. R., Fang, S., Palop, J. J., Yu, G.-Q., Wang, X., \& Mucke, L. (2008). Altered navigational strategy use and visuospatial deficits in hAPP transgenic mice. Neurobiology of Aging, 29(2), 253-266.

Easson, A. K., Fatima, Z., \& Mclntosh, A. R. (2019). Functional connectivity-based subtypes of individuals with and without autism spectrum disorder. Network Neuroscience (Cambridge, Mass.), 3(2), 344-362.

Engelhardt, L. E., Roe, M. A., Juranek, J., DeMaster, D., Paige Harden, K., Tucker-Drob, E. M., \& Church, J. A. (2017). Children's Head Motion During fMRI Tasks is Heritable and Stable over Time. 
Developmental Cognitive Neuroscience. https://doi.org/10.1016/j.dcn.2017.01.011

Falconer, D., \& Mackay, T. (1996). Introduction to Quantitative Genetics (4th edn). Investigative Genetics.

Feredoes, E., \& Postle, B. R. (2007). Localization of load sensitivity of working memory storage:

Quantitatively and qualitatively discrepant results yielded by single-subject and group-averaged approaches to fMRI group analysis. In Neurolmage (Vol. 35, Issue 2, pp. 881-903).

https://doi.org/10.1016/j.neuroimage.2006.12.029

Fonov, V., Coupe, P., Eskildsen, S., \& Collins, D. (2011). Atrophy-specific MRI brain template for Alzheimer's disease and mild cognitive impairment. Alzheimer's \& Dementia: The Journal of the Alzheimer's Association, 7(4, Supplement), S58.

Ganjgahi, H., Winkler, A. M., Glahn, D. C., Blangero, J., Kochunov, P., \& Nichols, T. E. (2015). Fast and powerful heritability inference for family-based neuroimaging studies. Neurolmage, 115, 256-268.

Giove, F., Gili, T., lacovella, V., Macaluso, E., \& Maraviglia, B. (2009). Images-based suppression of unwanted global signals in resting-state functional connectivity studies. Magnetic Resonance Imaging, 27(8), 1058-1064.

Glasser, M. F., Sotiropoulos, S. N., Wilson, J. A., Coalson, T. S., Fischl, B., Andersson, J. L., Xu, J., Jbabdi, S., Webster, M., Polimeni, J. R., Van Essen, D. C., Jenkinson, M., \& WU-Minn HCP Consortium. (2013). The minimal preprocessing pipelines for the Human Connectome Project. Neurolmage, 80, 105-124.

Grasby, K. L., Jahanshad, N., Painter, J. N., Colodro-Conde, L., Bralten, J., Hibar, D. P., Lind, P. A., Pizzagalli, F., Ching, C. R. K., McMahon, M. A. B., Shatokhina, N., Zsembik, L. C. P., Thomopoulos, S. I., Zhu, A. H., Strike, L. T., Agartz, I., Alhusaini, S., Almeida, M. A. A., Alnæs, D., ... Enhancing Neurolmaging Genetics through Meta-Analysis Consortium (ENIGMA)—Genetics working group. (2020). The genetic architecture of the human cerebral cortex. Science, 367(6484). https://doi.org/10.1126/science.aay6690

Grodd, W., Hülsmann, E., Lotze, M., Wildgruber, D., \& Erb, M. (2001). Sensorimotor mapping of the human cerebellum: fMRI evidence of somatotopic organization. Human Brain Mapping, 13(2), 
55-73.

Han, Y., \& Adolphs, R. (2019). Estimating the heritability of psychological measures in the Human Connectome Project dataset. bioRxiv. https://doi.org/10.1101/704023

Hasson, U., Landesman, O., Knappmeyer, B., Vallines, I., Rubin, N., \& Heeger, D. J. (2008).

Neurocinematics: The Neuroscience of Film. Current Population Reports. Series P-25, Population Estimates and Projections, 2(1), 1-26.

Heun, R., Jessen, F., Klose, U., Erb, M., Granath, D., Freymann, N., \& Grodd, W. (2000). Interindividual variation of cerebral activation during encoding and retrieval of words. European Psychiatry: The Journal of the Association of European Psychiatrists, 15(8), 470-479.

Hickok, G., \& Poeppel, D. (2007). The cortical organization of speech processing. Nature Reviews. Neuroscience, 8(5), 393-402.

Hodge, M. R., Horton, W., Brown, T., Herrick, R., Olsen, T., Hileman, M. E., McKay, M., Archie, K. A., Cler, E., Harms, M. P., Burgess, G. C., Glasser, M. F., Elam, J. S., Curtiss, S. W., Barch, D. M., Oostenveld, R., Larson-Prior, L. J., Ugurbil, K., Van Essen, D. C., \& Marcus, D. S. (2016). ConnectomeDB-Sharing human brain connectivity data. Neurolmage, 124(Pt B), 1102-1107. Kashyap, R., Bhattacharjee, S., Yeo, B. T. T., \& Chen, S. H. A. (2019). Maximizing dissimilarity in resting state detects heterogeneous subtypes in healthy population associated with high substance use and problems in antisocial personality. Human Brain Mapping. https://doi.org/10.1002/hbm.24873

Kherif, F., Poline, J.-B., Mériaux, S., Benali, H., Flandin, G., \& Brett, M. (2003). Group analysis in functional neuroimaging: selecting subjects using similarity measures. Neurolmage, 20(4), 2197-2208.

Kirchhoff, B. A., \& Buckner, R. L. (2006). Functional-anatomic correlates of individual differences in memory. Neuron, 51(2), 263-274.

Lebreton, M., \& Palminteri, S. (2016). Revisiting the assessment of inter-individual differences in fMRI activations-behavior relationships. bioRxiv. https://www.biorxiv.org/content/early/2016/04/11/036772.abstract

Le Guen, Y., Amalric, M., Pinel, P., Pallier, C., \& Frouin, V. (2018). Shared genetic aetiology between 
cognitive performance and brain activations in language and math tasks. Scientific Reports, 8(1), 17624.

Machielsen, W. C., Rombouts, S. A., Barkhof, F., Scheltens, P., \& Witter, M. P. (2000). FMRI of visual encoding: reproducibility of activation. Human Brain Mapping, 9(3), 156-164.

Maxwell, A. E., Fenwick, P. B. C., Fenton, G. W., \& Dollimore, J. (1974). Reading ability and brain function: a simple statistical model. In Psychological Medicine (Vol. 4, Issue 3, pp. 274-280). https://doi.org/10.1017/s0033291700042963

McGonigle, D. J., Howseman, A. M., Athwal, B. S., Friston, K. J., Frackowiak, R. S., \& Holmes, A. P. (2000). Variability in fMRI: an examination of intersession differences. Neurolmage, 11(6 Pt 1), 708-734.

Miller, M. B., Donovan, C.-L., Bennett, C. M., Aminoff, E. M., \& Mayer, R. E. (2012). Individual differences in cognitive style and strategy predict similarities in the patterns of brain activity between individuals. Neurolmage, 59(1), 83-93.

Miller, M. B., Donovan, C.-L., Van Horn, J. D., German, E., Sokol-Hessner, P., \& Wolford, G. L. (2009). Unique and persistent individual patterns of brain activity across different memory retrieval tasks. Neurolmage, 48(3), 625-635.

Miller, M. B., Van Horn, J. D., Wolford, G. L., Handy, T. C., Valsangkar-Smyth, M., Inati, S., Grafton, S., \& Gazzaniga, M. S. (2002). Extensive individual differences in brain activations associated with episodic retrieval are reliable over time. Journal of Cognitive Neuroscience, 14(8), 1200-1214.

Mugler, J. P., \& Brookeman, J. R. (1990). Three-dimensional magnetization-prepared rapid gradient-echo imaging (3D MP RAGE). Magnetic Resonance in Medicine: Official Journal of the Society of Magnetic Resonance in Medicine / Society of Magnetic Resonance in Medicine. http://onlinelibrary.wiley.com/doi/10.1002/mrm.1910150117/abstract

NIH Toolbox. (2015). http://www.healthmeasures.net/explore-measurement-systems/nih-toolbox Parasuraman, R., \& Jiang, Y. (2012). Individual differences in cognition, affect, and performance: behavioral, neuroimaging, and molecular genetic approaches. Neurolmage, 59(1), 70-82.

Pinel, P., \& Dehaene, S. (2013). Genetic and environmental contributions to brain activation during calculation. Neurolmage, 81, 306-316. 
Power, J. D., Barnes, K. A., Snyder, A. Z., Schlaggar, B. L., \& Petersen, S. E. (2012). Spurious but systematic correlations in functional connectivity MRI networks arise from subject motion. Neurolmage, 59(3), 2142-2154.

Rao, L.-L., Zhou, Y., Zheng, D., Yang, L.-Q., \& Li, S. (2018). Genetic Contribution to Variation in Risk Taking: A Functional MRI Twin Study of the Balloon Analogue Risk Task. Psychological Science, 29(10), 1679-1691.

Saliasi, E., Geerligs, L., Lorist, M. M., \& Maurits, N. M. (2014). Neural correlates associated with successful working memory performance in older adults as revealed by spatial ICA. PloS One, 9(6), e99250.

Seghier, M. L., Friston, K. J., \& Price, C. J. (2007). Detecting subject-specific activations using fuzzy clustering. Neurolmage, 36(3), 594-605.

Seghier, M. L., Lazeyras, F., Pegna, A. J., Annoni, J.-M., \& Khateb, A. (2008). Group analysis and the subject factor in functional magnetic resonance imaging: Analysis of fifty right-handed healthy subjects in a semantic language task. Human Brain Mapping, 29(4), 461-477.

Seghier, M. L., \& Price, C. J. (2009). Dissociating functional brain networks by decoding the between-subject variability. Neurolmage, 45(2), 349-359.

Smith, S. M., Nichols, T. E., Vidaurre, D., Winkler, A. M., Behrens, T. E. J., Glasser, M. F., Ugurbil, K., Barch, D. M., Van Essen, D. C., \& Miller, K. L. (2015). A positive-negative mode of population covariation links brain connectivity, demographics and behavior. Nature Neuroscience, 18(11), $1565-1567$.

Sodini, S. M., Kemper, K. E., Wray, N. R., \& Trzaskowski, M. (2018). Comparison of Genotypic and Phenotypic Correlations: Cheverud's Conjecture in Humans. Genetics, 209(3), 941-948.

Stromswold, K. (2001). The Heritability of Language: A Review and Metaanalysis of Twin, Adoption, and Linkage Studies. Language, 77(4), 647-723.

Sunkin, S. M., Ng, L., Lau, C., Dolbeare, T., Gilbert, T. L., Thompson, C. L., Hawrylycz, M., \& Dang, C. (2013). Allen Brain Atlas: an integrated spatio-temporal portal for exploring the central nervous system. Nucleic Acids Research, 41(Database issue), D996-D1008.

Urchs, S. G., Tam, A., Orban, P., Moreau, C., Benhajali, Y., Nguyen, H. D., Evans, A. C., \& Bellec, P. 
(2020). Subtypes of functional connectivity associate robustly with ASD diagnosis. In bioRxiv (p. 2020.04.14.040576). https://doi.org/10.1101/2020.04.14.040576

van der Meulen, M., Steinbeis, N., Achterberg, M., van IJzendoorn, M. H., \& Crone, E. A. (2018).

Heritability of neural reactions to social exclusion and prosocial compensation in middle childhood.

Developmental Cognitive Neuroscience, 34, 42-52.

Van Essen, D. C., Smith, S. M., Barch, D. M., Behrens, T. E. J., Yacoub, E., Ugurbil, K., \& WU-Minn HCP Consortium. (2013). The WU-Minn Human Connectome Project: an overview. Neurolmage, $80,62-79$.

Van Horn, J. D., Grafton, S. T., \& Miller, M. B. (2008). Individual Variability in Brain Activity: A Nuisance or an Opportunity? Brain Imaging and Behavior, 2(4), 327-334.

Worsley, K. J., Liao, C., Grabove, M., Petre, V., Ha, B., \& Evans, A. C. (2003). A General Statistical Analysis for fMRI Data. http://www.math.mcgill.ca/keith/HBM2000/Poster.ppt

Yaple, Z. A., Stevens, W. D., \& Arsalidou, M. (2019). Meta-analyses of the n-back working memory task: fMRI evidence of age-related changes in prefrontal cortex involvement across the adult lifespan. Neurolmage, 196, 16-31.

Zhang, X., Mormino, E. C., Sun, N., Sperling, R. A., Sabuncu, M. R., Yeo, B. T. T., Weiner, M. W., Aisen, P., Weiner, M., Petersen, R., \& Others. (2016). Bayesian model reveals latent atrophy factors with dissociable cognitive trajectories in Alzheimer's disease. Proceedings of the National Academy of Sciences, 113(42), E6535-E6544.

\section{Supplementary material}




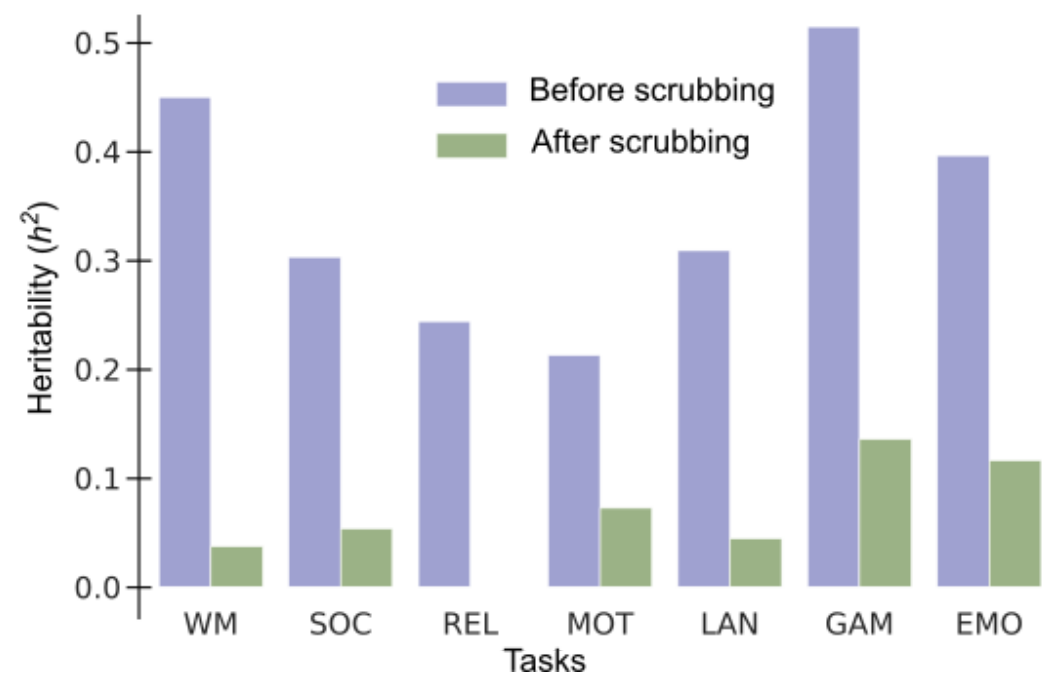

Figure Sup1 - Heritability of framewise displacement (FD) measure before and after scrubbing on the 7 tasks. WM: working memory, SOC: social, REL: relational, MOT: motor, LAN: language, GAM: gambling and EMO: emotional. Heritability of FD was estimated before and after scrubbing to shows how motion reduction used in this paper is efficient in reducing the heritability estimate that is known to affect further estimate of heritability on brain phenotypes.

\begin{tabular}{|c|c|c|}
\hline columnHeader & assessment & fullDisplayName \\
\hline EmoAcc & Emotion & OVERALL Emotion Task accuracy \\
\hline EmoRT & Emotion & $\begin{array}{l}\text { OVERALL Emotion Task Reaction } \\
\text { Time }\end{array}$ \\
\hline GamRT & Gambling & $\begin{array}{l}\text { Gambling Task Overall Reaction } \\
\text { Time 'Larger' }\end{array}$ \\
\hline LanAcc & Language & Language Task OVERALL accuracy \\
\hline LanRT & Language & $\begin{array}{l}\text { Language Task OVERALL median } \\
\text { Reaction Time }\end{array}$ \\
\hline RelAcc & Relational & Relational Task OVERALL accuracy \\
\hline RelRT & Relational & $\begin{array}{l}\text { Relational Task OVERALL Reaction } \\
\text { Time }\end{array}$ \\
\hline SocRand & Social & $\begin{array}{l}\text { Social Task Percentage 'Random' in } \\
\text { Random condition }\end{array}$ \\
\hline SocRRT & Social & $\begin{array}{l}\text { Social Task Median Reaction Time } \\
\text { 'Random' in Random condition }\end{array}$ \\
\hline SocTOM & Social & $\begin{array}{l}\text { Social Task Percentage 'TOM' in } \\
\text { Social (TOM) condition }\end{array}$ \\
\hline SocTRT & Social & $\begin{array}{l}\text { Social Task Median Reaction Time } \\
\text { 'TOM' in Social (TOM) condition }\end{array}$ \\
\hline WM2bkAcc & Working Memory & $\begin{array}{l}\text { Working Memory Task Accuracy for } \\
\text { 2-back }\end{array}$ \\
\hline WM2bkRT & Working Memory & $\begin{array}{l}\text { Working Memory Task Median } \\
\text { Reaction Time for 2-back }\end{array}$ \\
\hline WMObkAcc & Working Memory & $\begin{array}{l}\text { Working Memory Task Accuracy for } \\
\text { 0-back }\end{array}$ \\
\hline WMObkRT & Working Memory & $\begin{array}{l}\text { Working Memory Task Median } \\
\text { Reaction Time for 0-back }\end{array}$ \\
\hline
\end{tabular}

Table 1 - In-scanner task performance description- In-scanner accuracy and reaction time measures used in this study. 


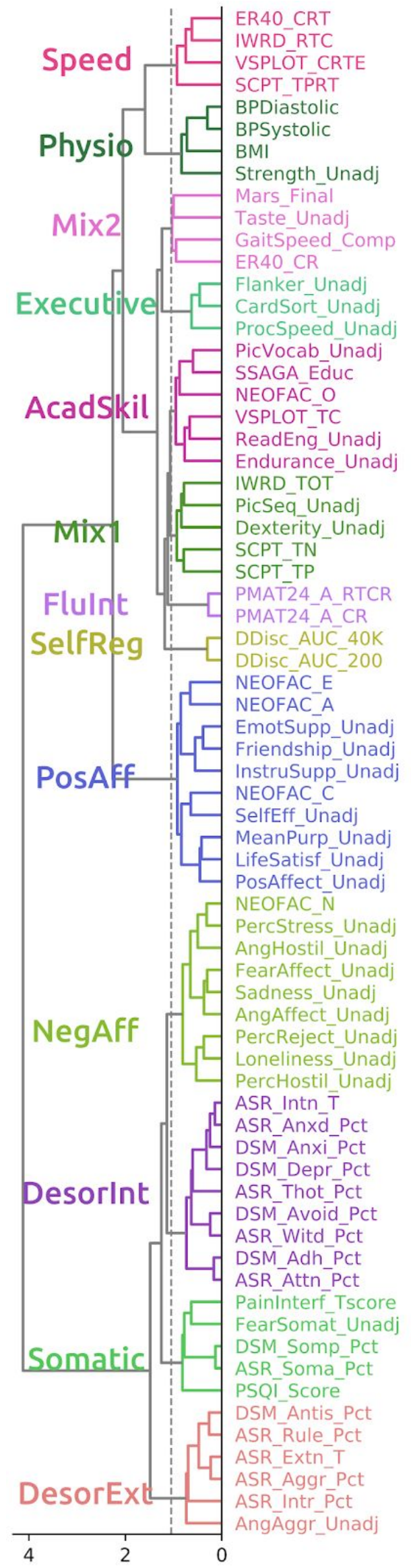

Emotion Recognition Penn Emotion Recognition Test: Correct Responses Median Verbal Episodic Memory Penn Word Memory Test: Median Reaction Time for Correct Responses Spatial Orientation Penn Line Orientation: Median Reaction Time

Sustained Attention Short Penn Continuous Performance Test: Median Response Time Physical Health Diastolic blood pressure of subject

Physical Health Systolic blood pressure of subject

Physical Health Body Mass Index

Grip Strength Dynamometry NIH Toolbox Grip Strength Test: Unadjusted Scale Score

Contrast Sensitivity Mars Final Contrast Sensitivity Score

NIH Toolbox Regional Taste Intensity Age 12+ Unadjusted Scale Score

Locomotion NIH Toolbox 4-Meter Walk Gait Speed Test: Computed Score

Penn Emotion Recognition Test: Number of Correct Responses

Executive Function NIH Toolbox Flanker Inhibitory Control and Attention Test: Unadjusted Scale Executive Function NIH Toolbox Dimensional Change Card Sort Test: Unadjusted Scale Score Processing Speed NIH Toolbox Pattern Comparison Processing Speed Test: Unadjusted Scale Language/Vocabulary Comprehension NIH Toolbox Picture Vocabulary: Unadjusted Scale Score Demographics Years of education completed

Personality Five Factor Model NEO-FFI Openness to Experience Spatial Orientation Penn Line Orientation: Total Number Correct

Language/Reading Decoding NIH Toolbox Oral Reading Recognition Test: Unadjusted Scale Score Endurance NIH Toolbox 2-minute Walk Endurance Test: Unadjusted Scale Score Verbal Episodic Memory Penn Word Memory Test: Total Number of Correct Responses Episodic Memory NIH Toolbox Picture Sequence Memory Test: Unadjusted Scale Score Dexterity NIH Toolbox 9-hole Pegboard Dexterity Test: Unadjusted Scale Score Sustained Attention Short Penn Continuous Performance Test: True Negatives Sustained Attention Short Penn Continuous Performance Test: True Positives Fluid Intelligence Penn Progressive Matrices: Median Reaction Time for Correct Responses Fluid Intelligence Penn Progressive Matrices: Number of Correct Responses Self-regulation/Impulsivity Delay Discounting: Area Under the Curve for Discounting of $\$ 40,00$ Self-regulation/Impulsivity Delay Discounting: Area Under the Curve for Discounting of $\$ 200$ Personality Five Factor Model NEO-FFI Extroversion

Personality Five Factor Model NEO-FFI Agreeableness

Social Relationships NIH Toolbox Emotional Support Survey: Unadjusted Scale Score Social Relationships NIH Toolbox Friendship Survey: Unadjusted Scale Score Social Relationships NIH Toolbox Instrumental Support Survey: Unadjusted Scale Score Personality Five Factor Model NEO-FFI Conscientiousness

Stress and Self Efficacy NIH Toolbox Self-Efficacy Survey: Unadjusted Scale Score

Psychological Well-being NIH Toolbox Meaning and Purpose Survey: Unadjusted Scale Score Psychological Well-being NIH Toolbox General Life Satisfaction Survey: Unadjusted Scale Score Psychological Well-being NIH Toolbox Positive Affect Survey: Unadjusted Scale Score Personality Five Factor Model NEO-FFI Neuroticism

Stress and Self Efficacy NIH Toolbox Perceived Stress Survey: Unadjusted Scale Score Negative Affect NIH Toolbox Anger-Hostility Survey: Unadjusted Scale Score

Negative Affect NIH Toolbox Fear-Affect Survey: Unadjusted Scale Score Negative Affect NIH Toolbox Sadness Survey: Unadjusted Scale Score

Negative Affect NIH Toolbox Anger-Physical Aggression Survey: Unadjusted Scale Score Social Relationships NIH Toolbox Perceived Rejection Survey: Unadjusted Scale Score Social Relationships NIH Toolbox Loneliness Survey: Unadjusted Scale Score Social Relationships NIH Toolbox Perceived Hostility Survey: Unadjusted Scale Score Life Function ASR Intrusive Raw Score Life Function ASR Anxious/Depressed Raw Score Life Function ASR DSM Anxiety Problems Raw Score Life Function ASR DSM Depressive Problems Raw Score Life Function ASR Thought Problems Raw Score Life Function ASR DSM Avoidant Personality Problems Raw Score Life Function ASR Withdrawn Raw Score Life Function ASR DSM AD/H Problems Raw Score Life Function ASR Attention Problems Raw Score Pain Intensity and Interference Surveys NIH Toolbox Pain Interference Survey Age 18+: T-score Negative Affect NIH Toolbox Fear-Somatic Arousal Survey: Unadjusted Scale Score

Life Function ASR DSM Somatic Problems Raw Score Life Function ASR Somatic Complaints Raw Score

Pittsburgh Sleep Quality Index Life Function ASR DSM Antisocial Personality Problems Raw Score Life Function ASR Rule Breaking Behavior Raw Score Life Function ASR Externalizing Raw Score

Life Function ASR Aggressive Behavior Raw Score Life Function ASR Intrusive Raw Score

Negative Affect NIH Toolbox Anger-Physical Aggression Survey: Unadjusted Scale Score

Figure Sup2 - Behavioral measures clusters. Group activation maps for motor and language tasks. 74 behavioral test are clustered using k-means. The dendrogram show 13 clusters cut off that are Speed: task reaction time measures, Physio: physiological measures, Mix2 and Mix1: a mixed categories of measures, Executive: working memory and cognitive flexibility measures, AcadSkil: language and vocabulary test along with 
academic completion measure, Flulnt: fluid intelligence measures, SelfReg: working memory and cognitive flexibility measures, PosAff: positive affect measures, NegAff: negative affect measures, Disorlnt: internalized disorders, Somatic: symptoms, problems or sensory manifestation related to the body, DisorExt: dysregulated externalized behaviour.
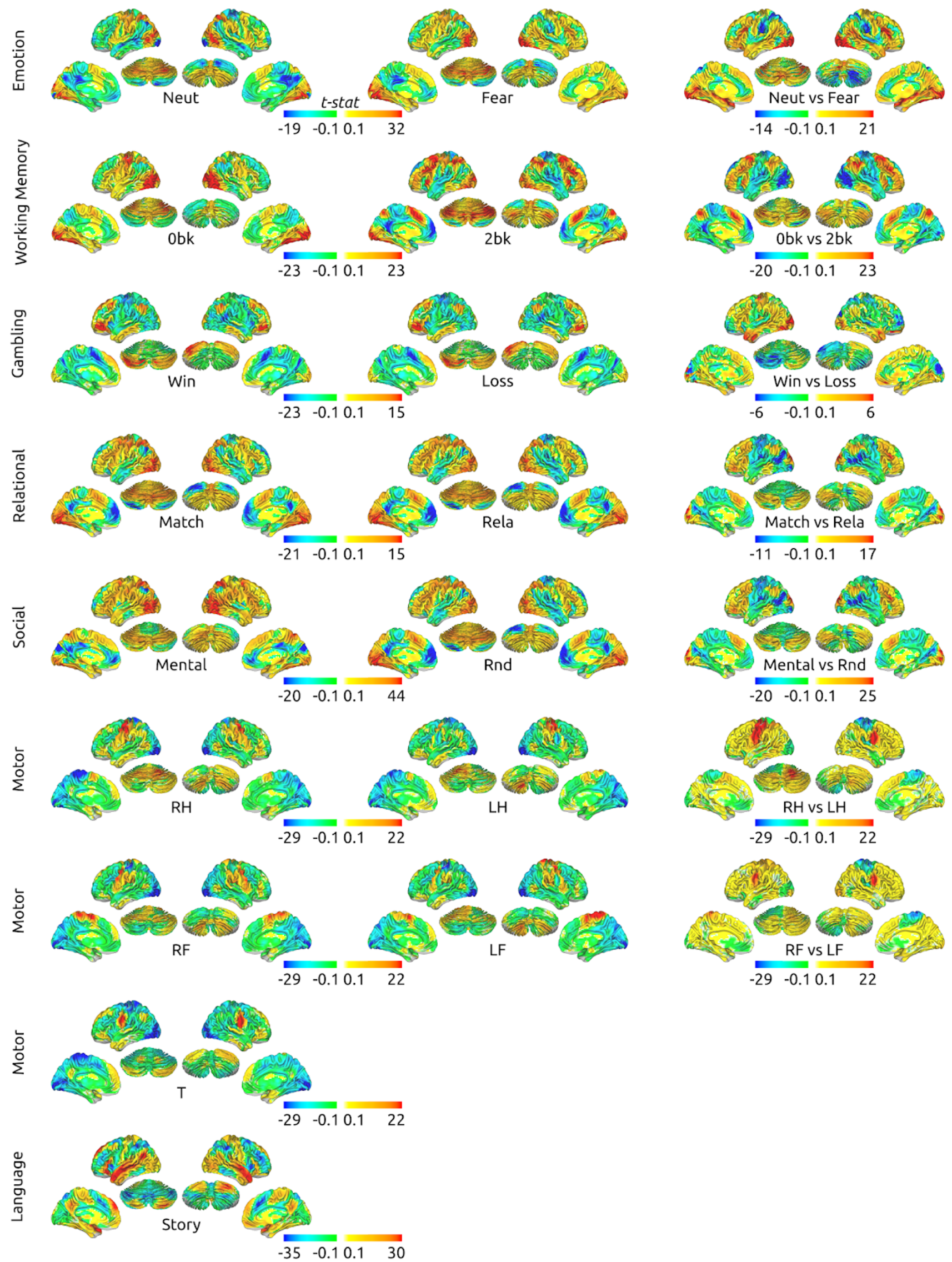
Figure Sup3 - Group activation maps for the 7 HCP tasks. Emotion task: Group activation maps for neutral condition (Neut), fear condition (Fear) and the contrast Neut vs Fear. Working memory task: Group activation maps for 0-back (0bk), 2-back (2bk) and the contrast Obk vs 2bk. Gambling task: Group activation maps for win (Win), loss (Loss) and the contrast Win vs Loss. Relational task: Group activation maps for Match, relational (Rel) and the contrast Match vs Rel. Social task: Group activation maps for Mental, random (Rnd) and the contrast Mental vs Rnd. Motor task: Group activation maps for left hand (LH), right hand (RH), left foot (LF), right foot (RF), tongue (T), contrast RF vs LF and contrast RH vs LH. Language task: Group activation maps for story.
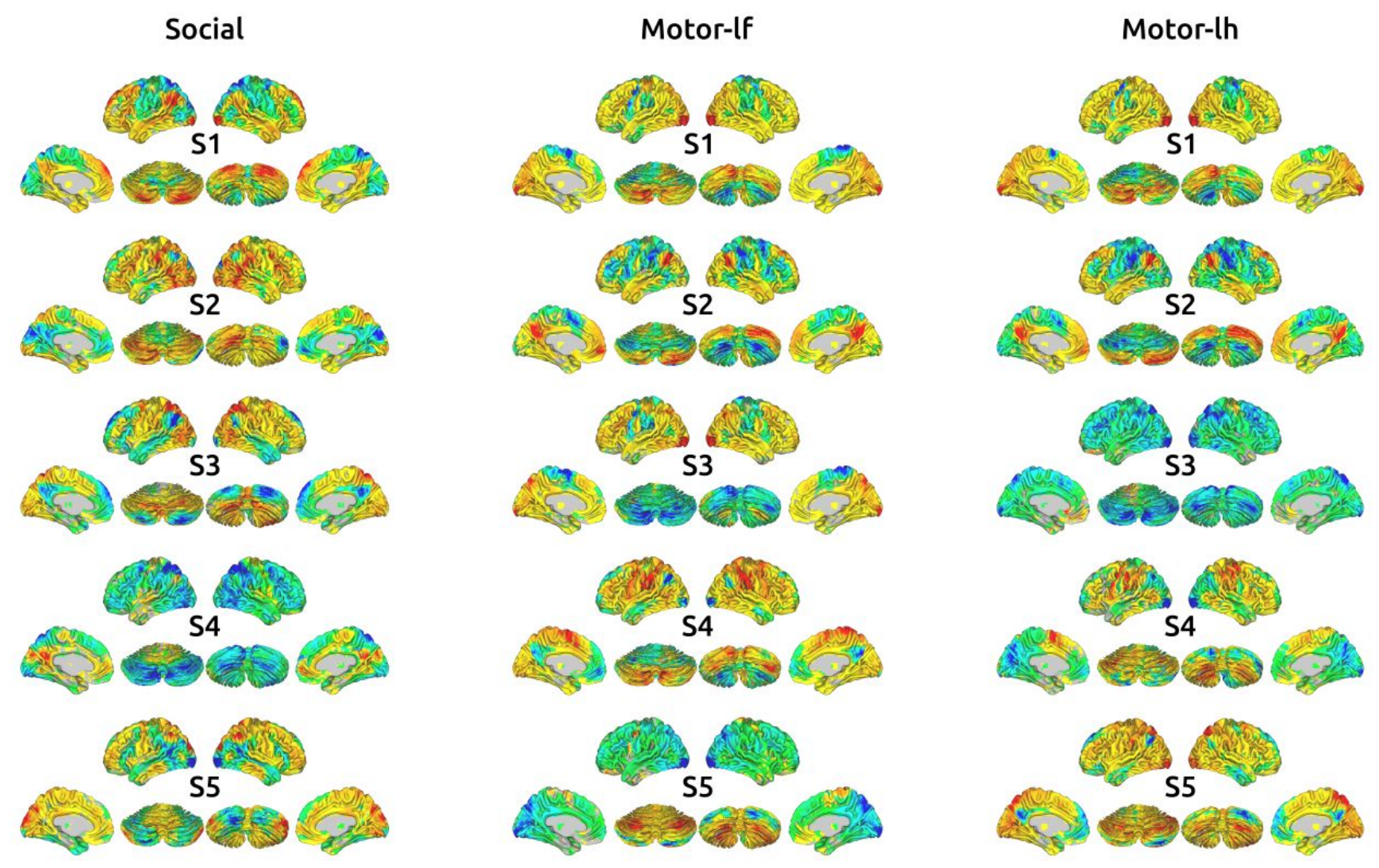


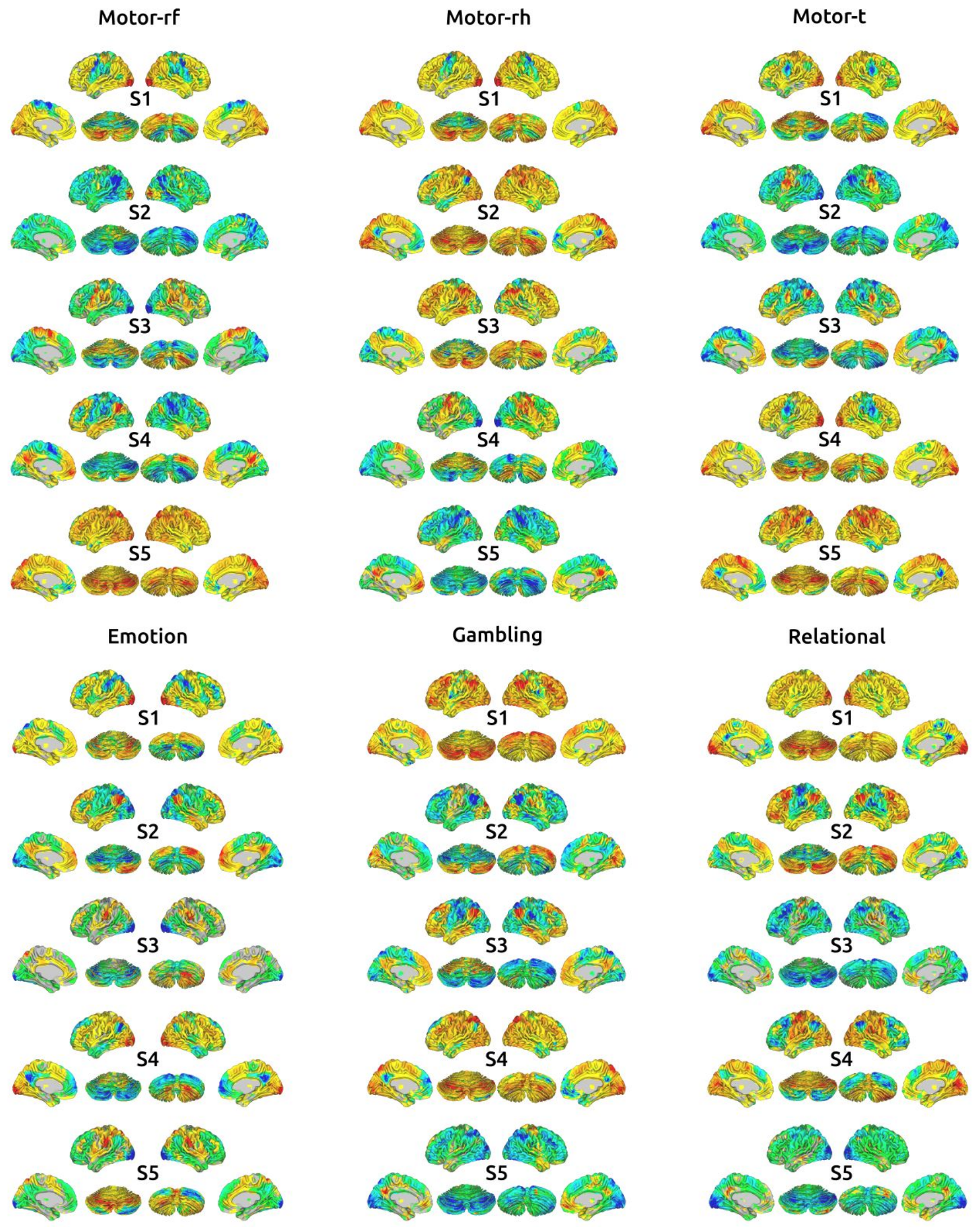

Figure Sup4 - Task activation subtypes: Fives subtypes maps (S1 to S5) for each of the social, motor, emotion, gambling and relational tasks. Working memory and language subtypes are shown on the manuscript figure 3. 
rf Gen Corr Matrix

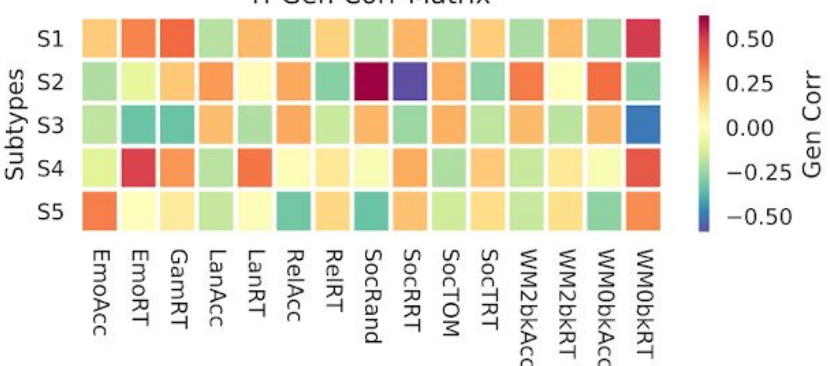

In-scanner Task Performance

rh Gen Corr Matrix

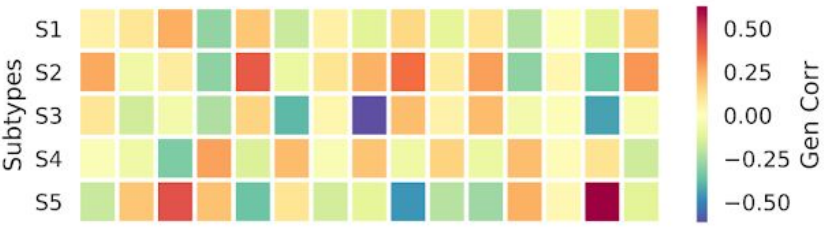

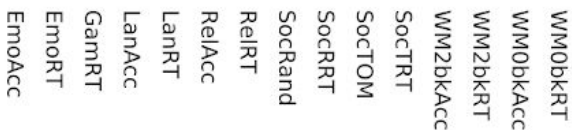

In-scanner Task Performance

t Gen Corr Matrix

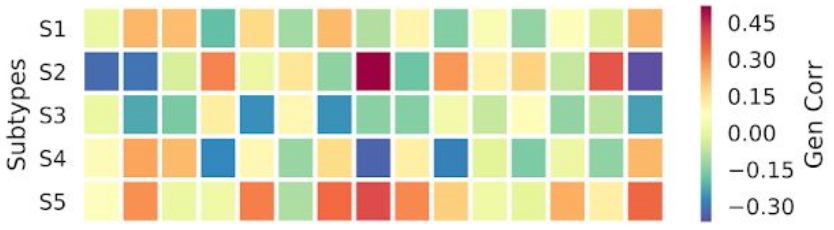

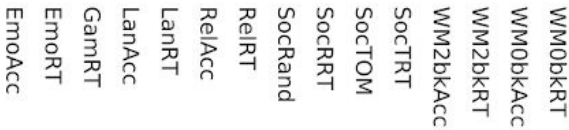

In-scanner Task Performance

contrast_fear_vs_neut Gen Corr Matrix

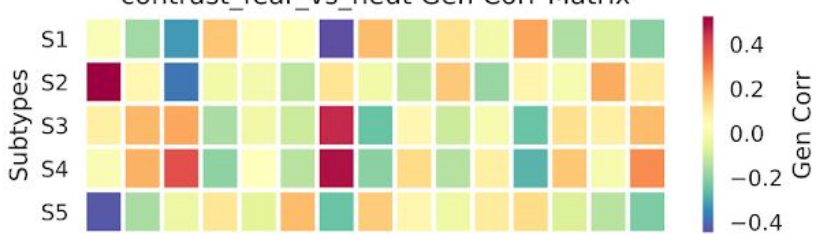

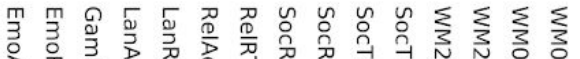

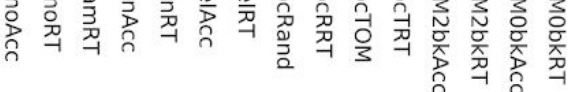

In-scanner Task Performance

contrast_win_vs_loss Gen Corr Matrix

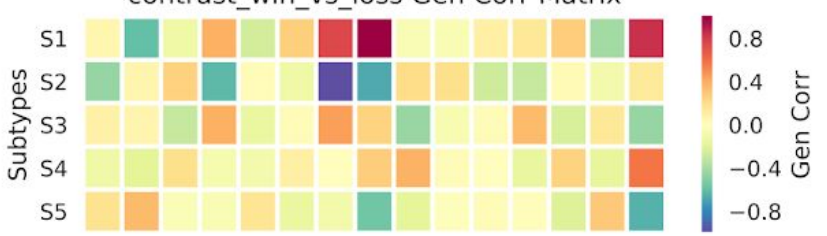

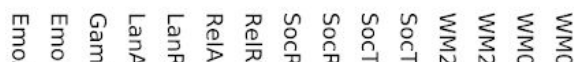

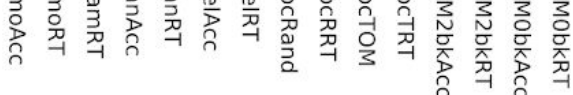

In-scanner Task Performance rf Pheno Corr Matrix

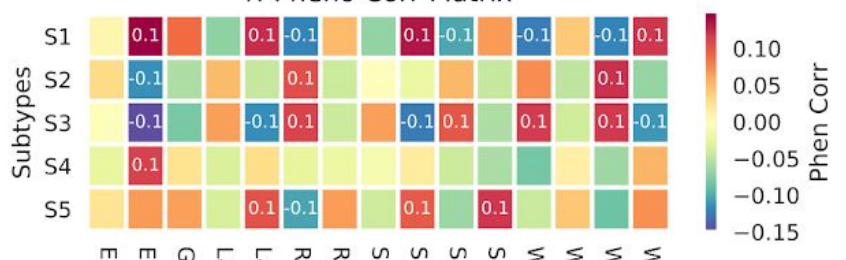

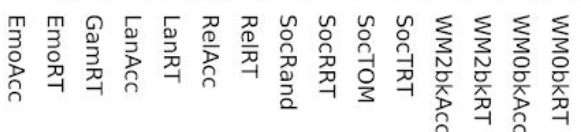

In-scanner Task Performance

rh Pheno Corr Matrix

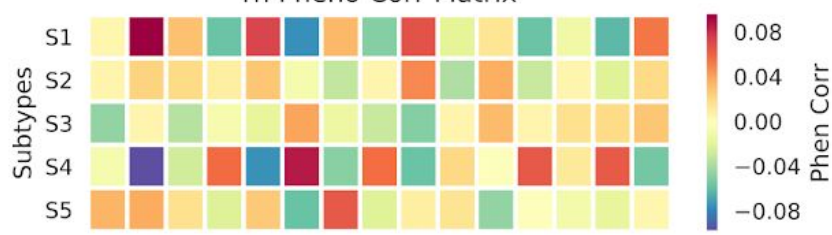

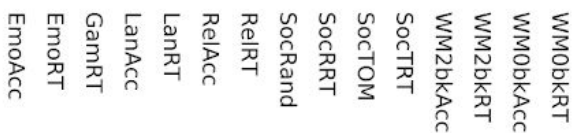

In-scanner Task Performance

t Pheno Corr Matrix

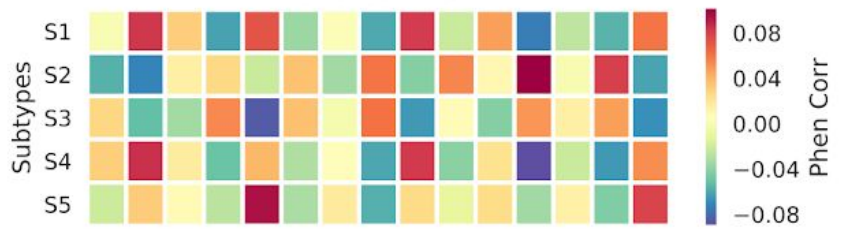

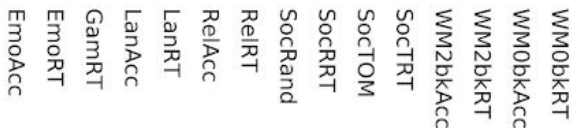

In-scanner Task Performance

contrast_fear_vs_neut Pheno Corr Matrix

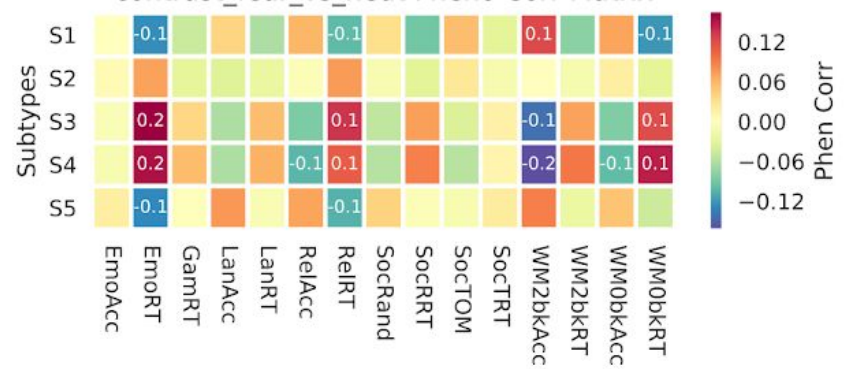

In-scanner Task Performance

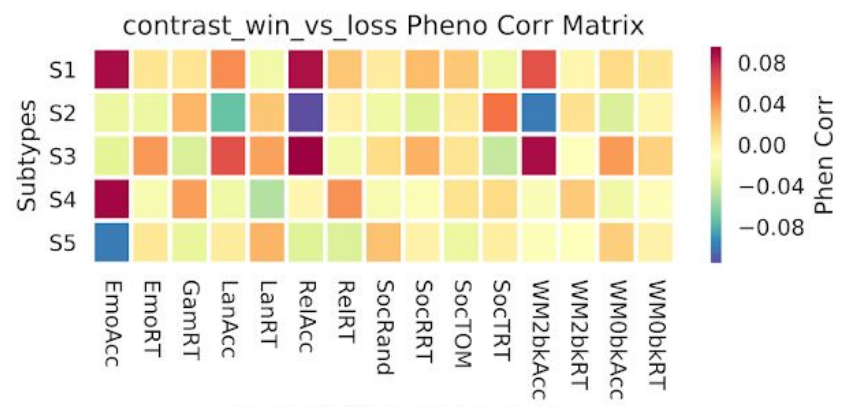

In-scanner Task Performance 


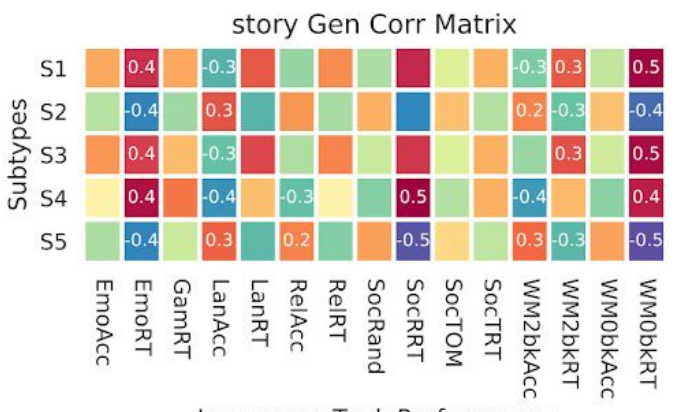

In-scanner Task Performance

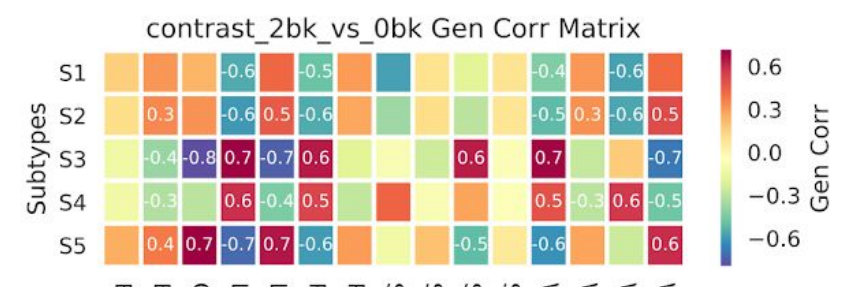

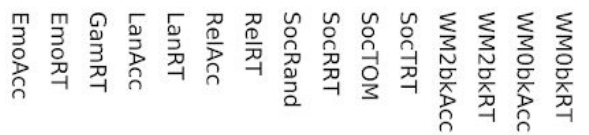

In-scanner Task Performance

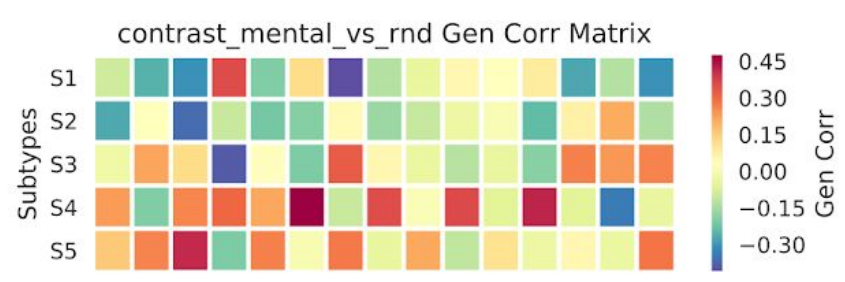

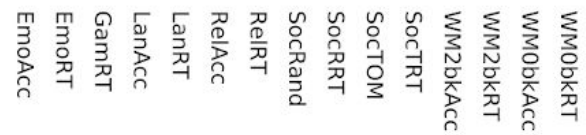

In-scanner Task Performance

If Gen Corr Matrix

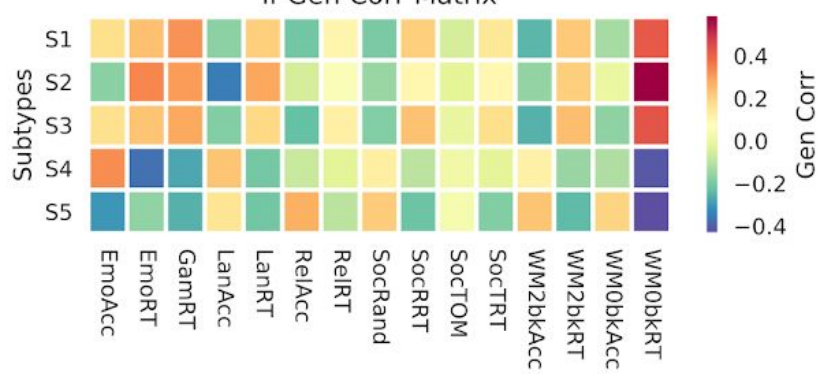

In-scanner Task Performance

Ih Gen Corr Matrix

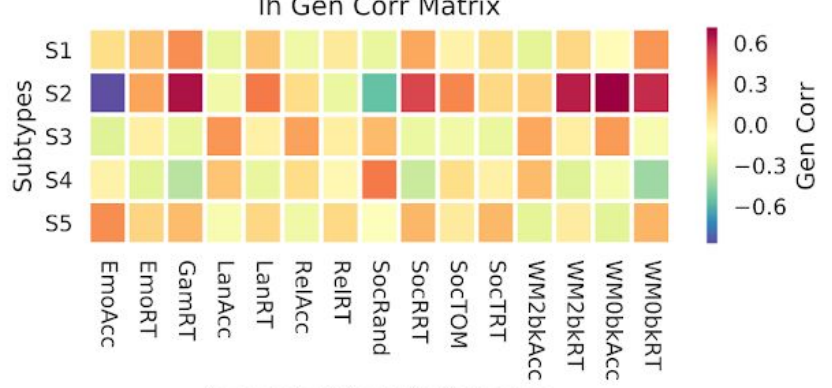

In-scanner Task Performance story Pheno Corr Matrix

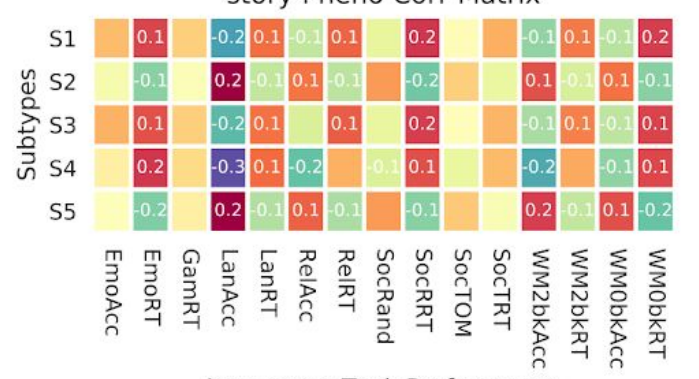

In-scanner Task Performance

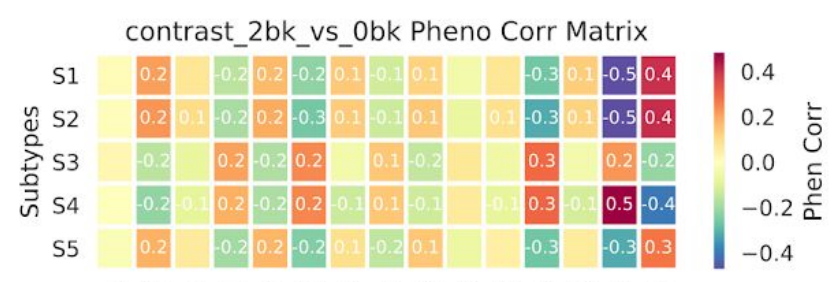

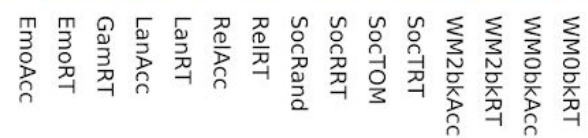

In-scanner Task Performance

contrast_mental_vs_rnd Pheno Corr Matrix

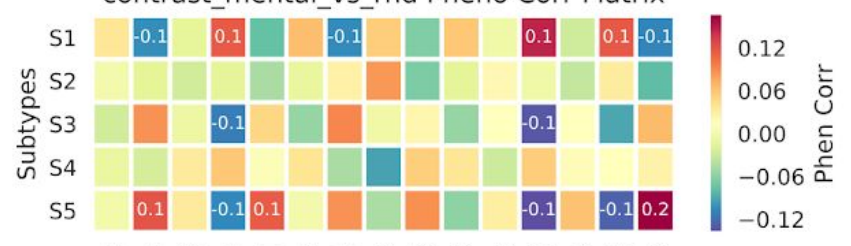

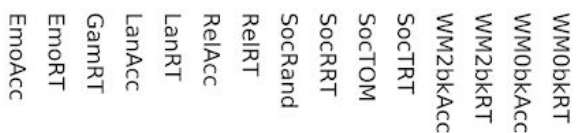

In-scanner Task Performance

If Pheno Corr Matrix

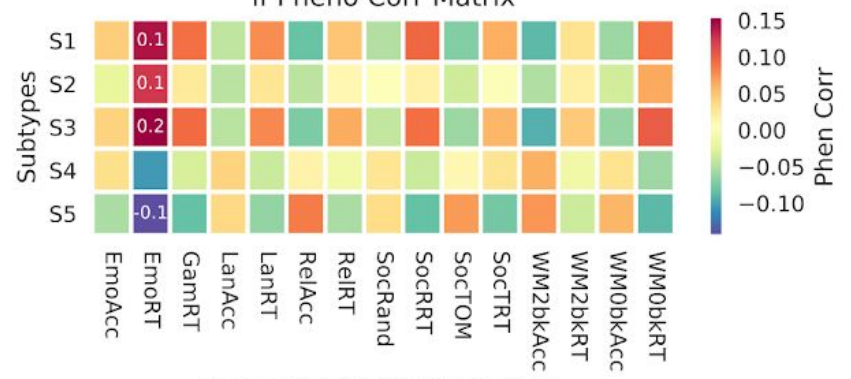

In-scanner Task Performance

Ih Pheno Corr Matrix

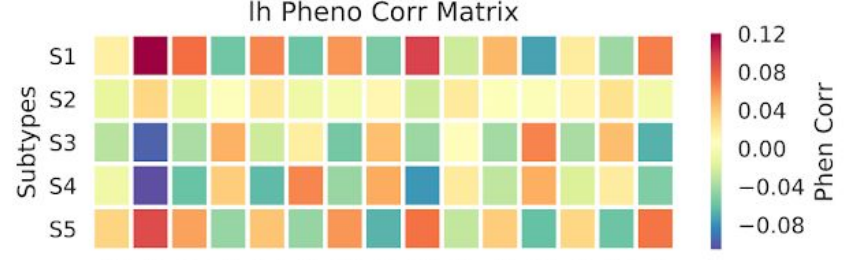

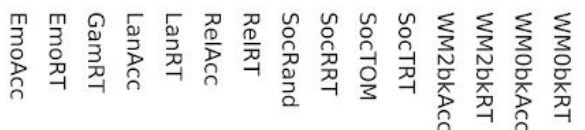

In-scanner Task Performance 

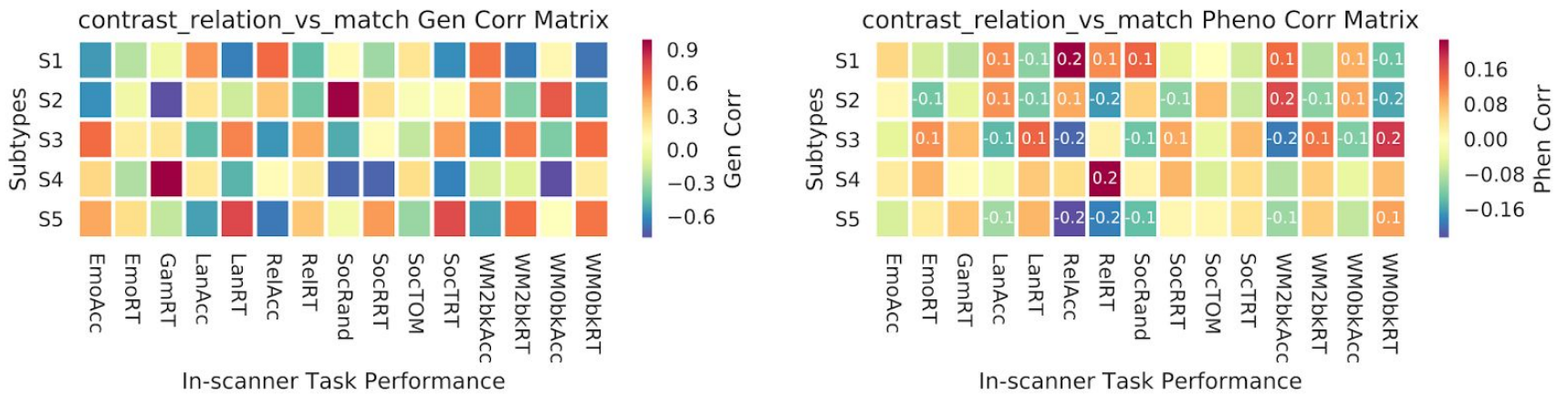

Figure Supp 5 - Genetic and phenotypic correlation for in-scanner behavioral performance: Left column represents genetic correlation between in scanner behavioral performance and brain subtypes weights. Right column represents phenotypic correlation between in scanner behavioral performance and brain subtypes weights. 


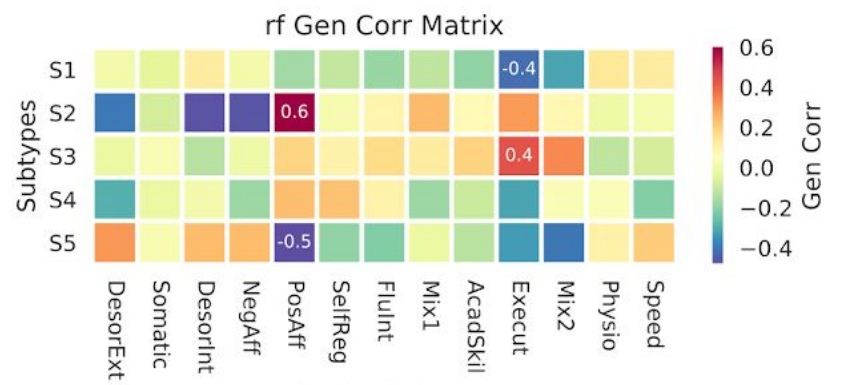

Behavioral Clusters

rh Gen Corr Matrix
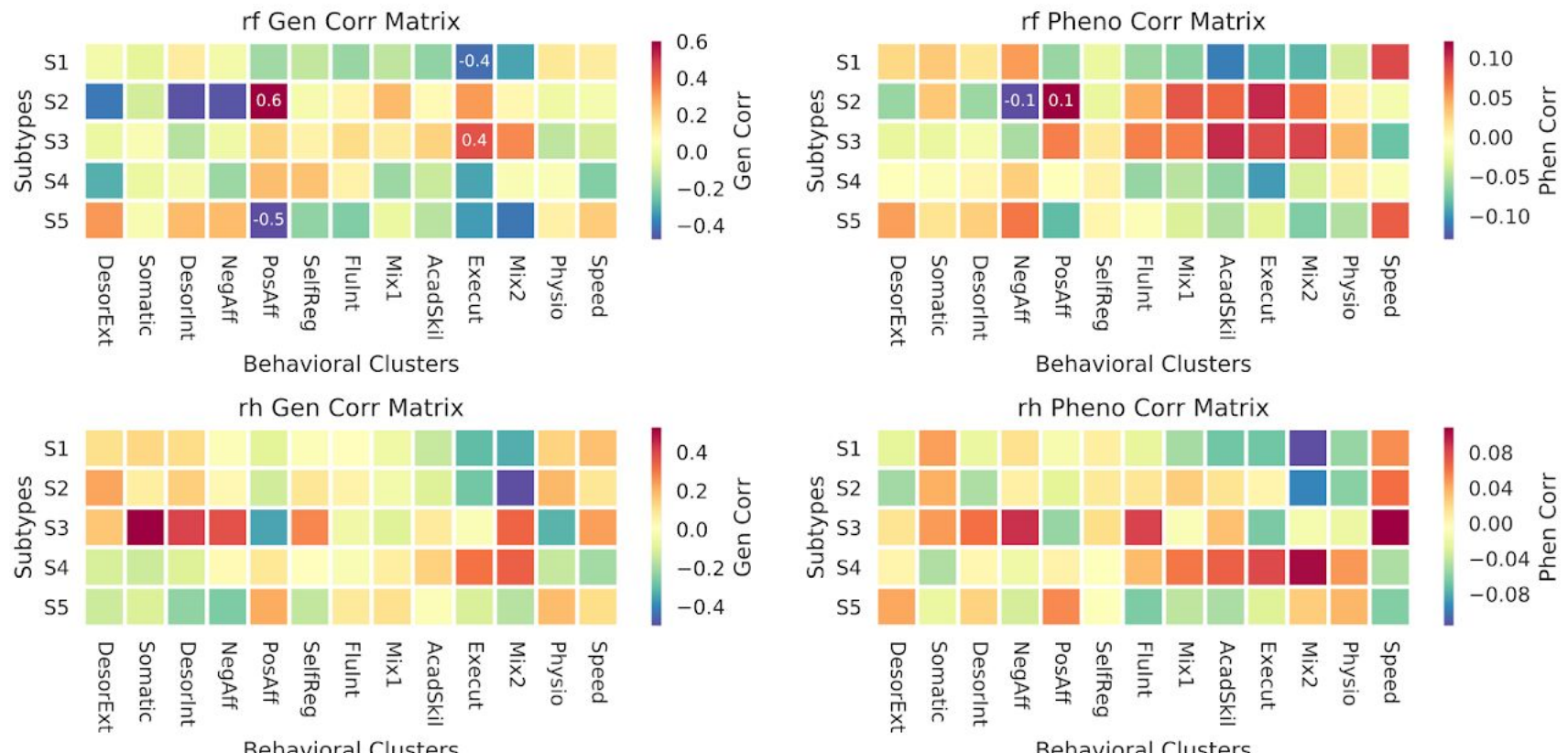

Behavioral Clusters
rh Pheno Corr Matrix

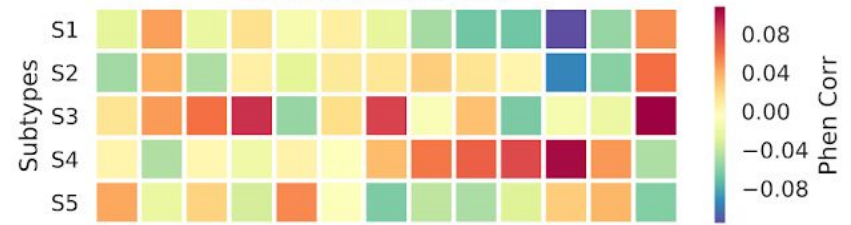

t Gen Corr Matrix

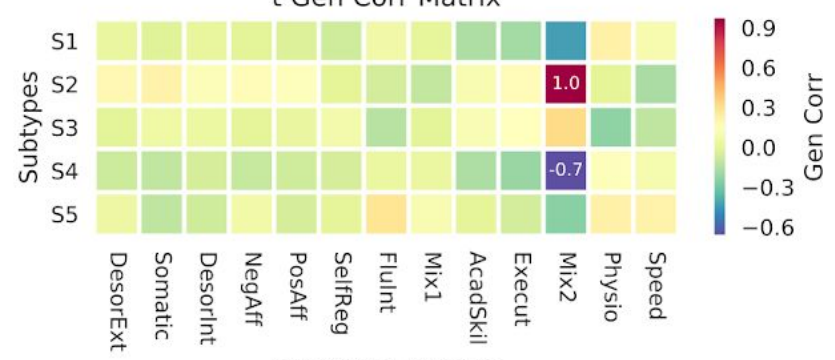

Behavioral Clusters

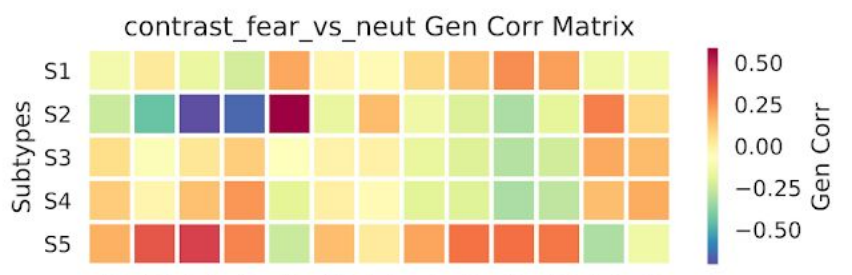

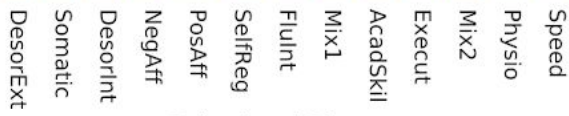

Behavioral Clusters

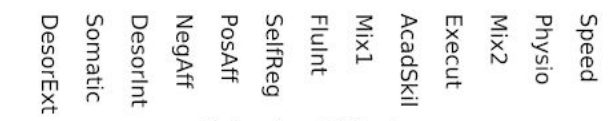

Behavioral Clusters

t Pheno Corr Matrix
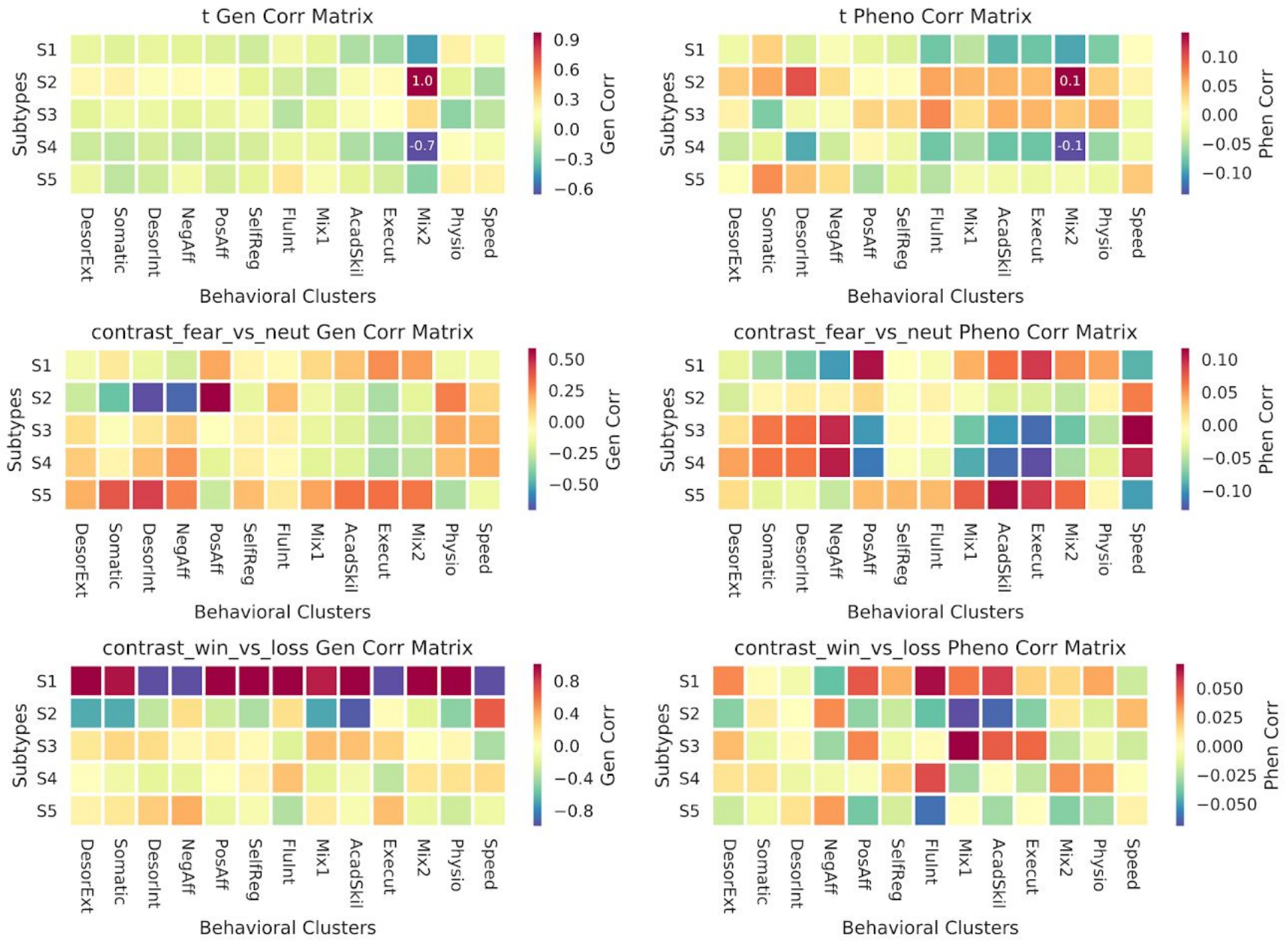
story Gen Corr Matrix

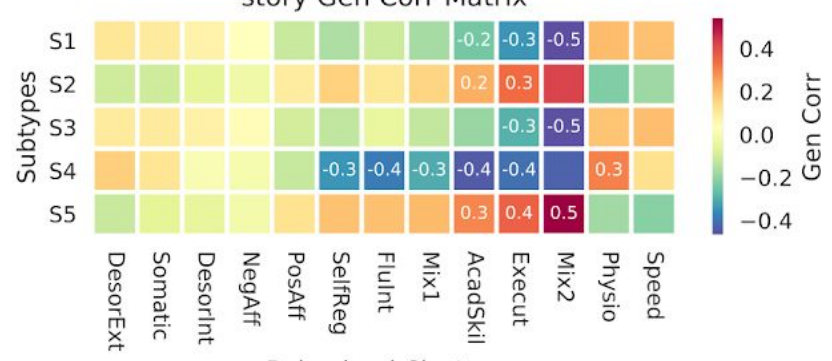

Behavioral Clusters
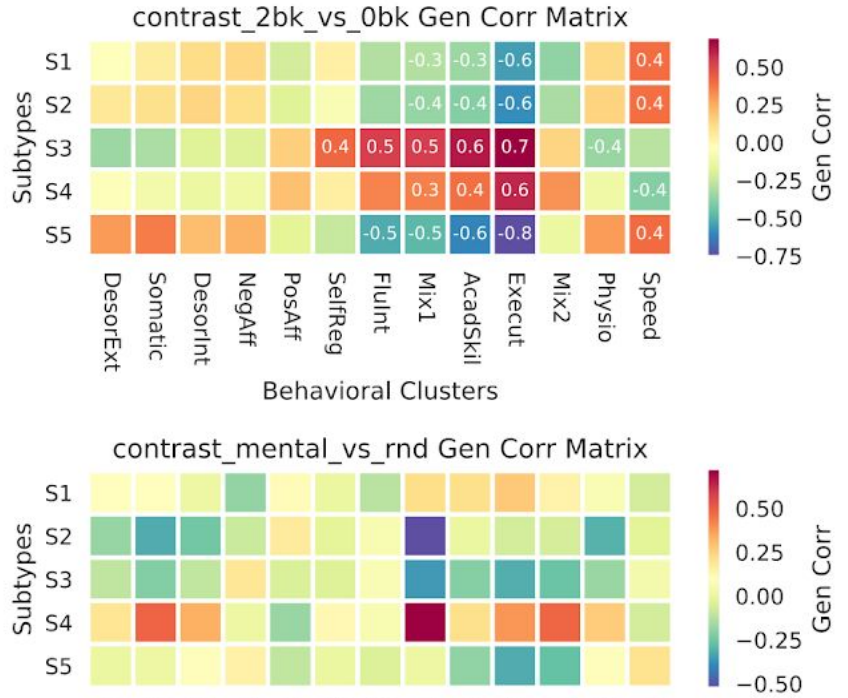

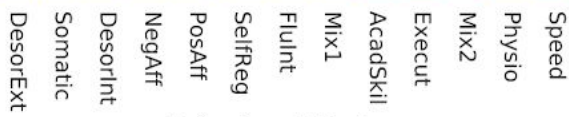

Behavioral Clusters

If Gen Corr Matrix

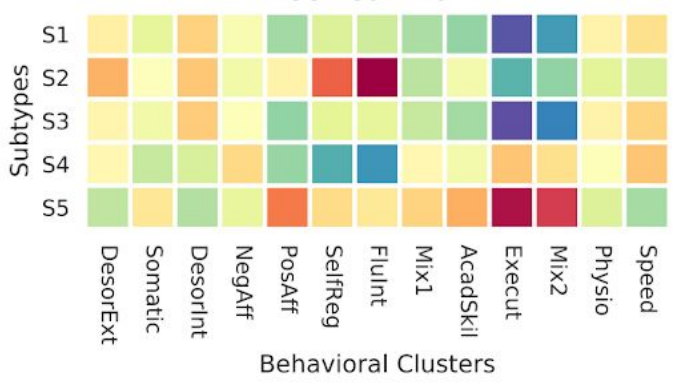

Ih Gen Corr Matrix
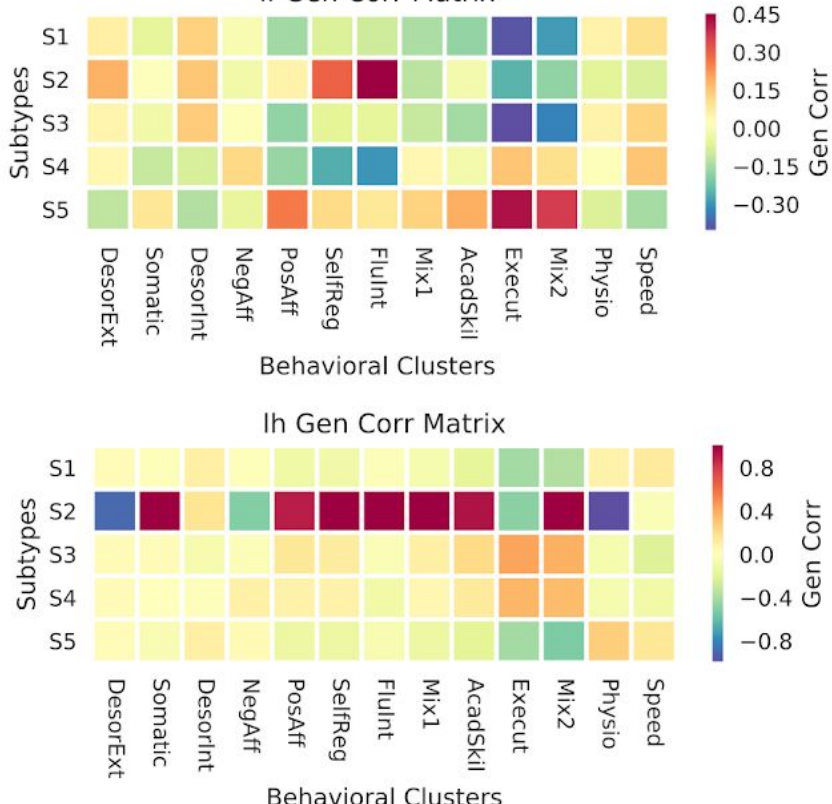

story Pheno Corr Matrix

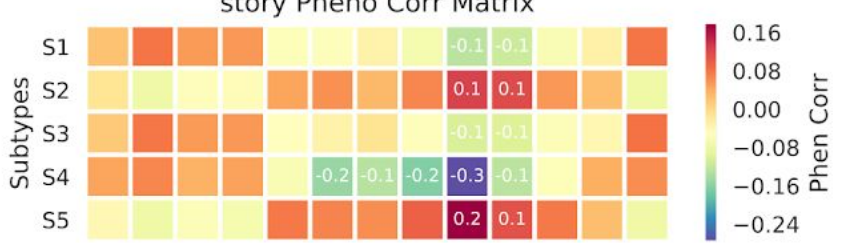

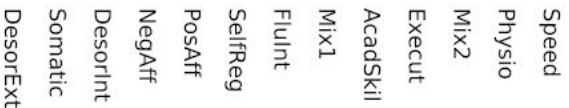

Behavioral Clusters

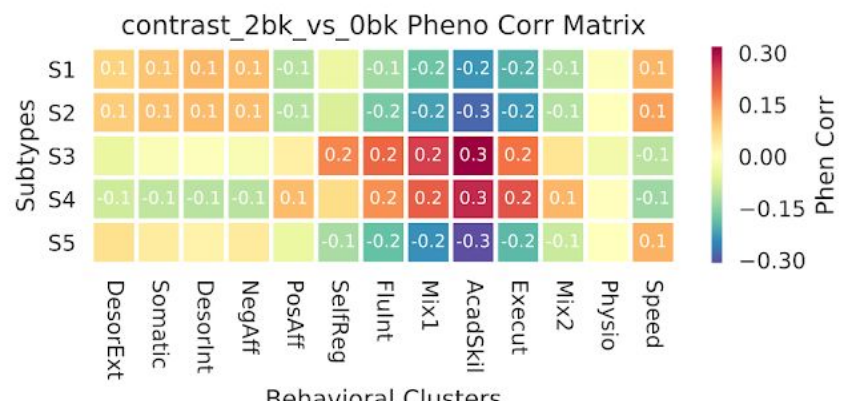

Behavioral Clusters

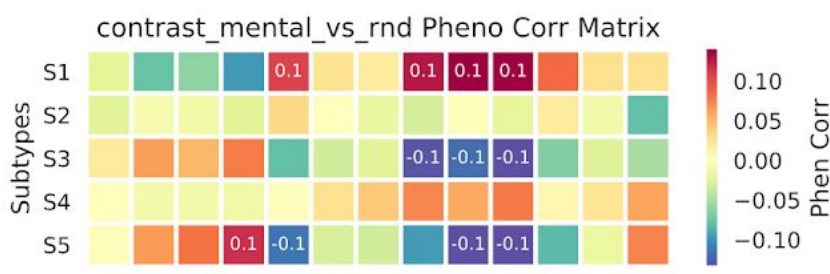

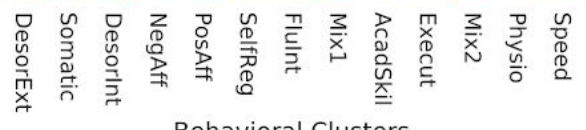

Behavioral Clusters

If Pheno Corr Matrix

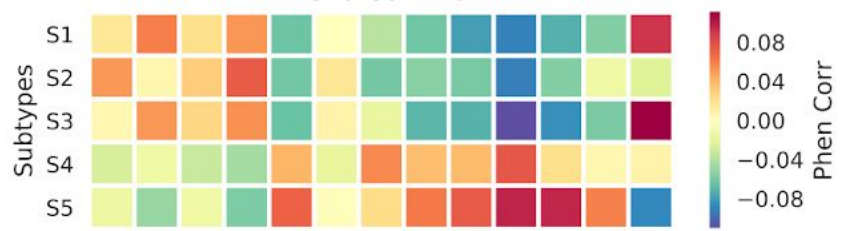

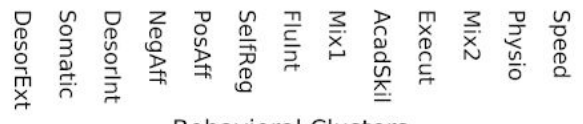

Behavioral Clusters

Ih Pheno Corr Matrix

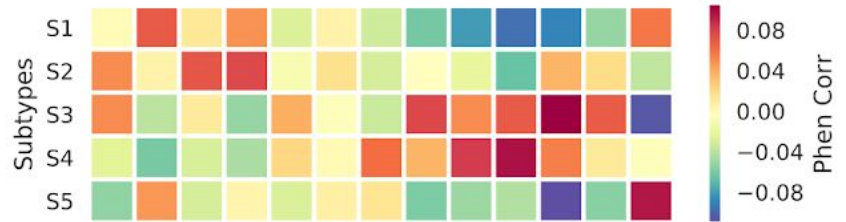

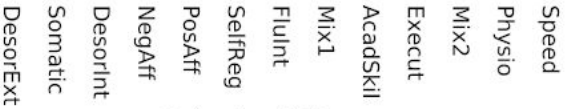
Behavioral Clusters 

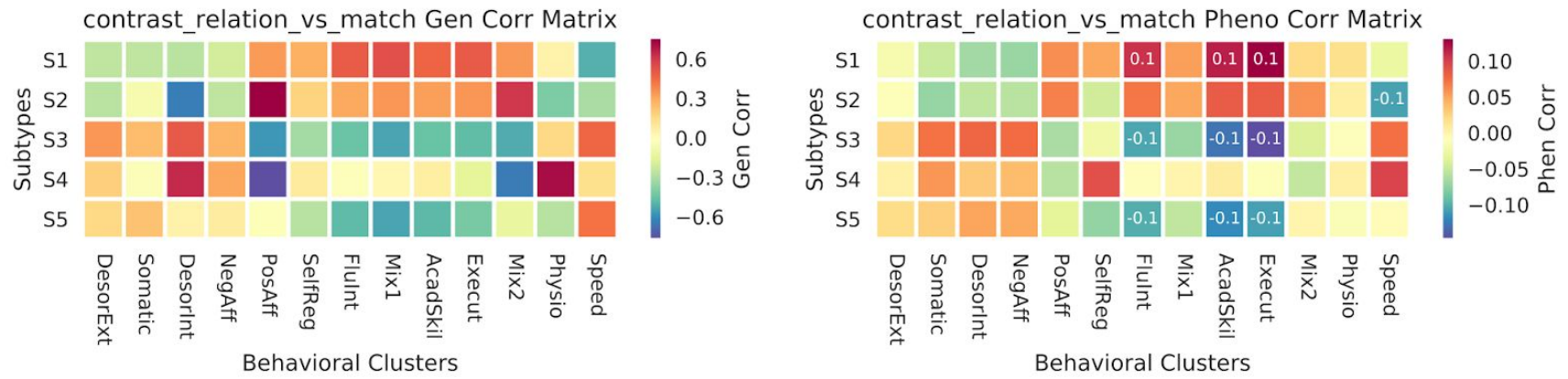

Figure Supp 6 - Genetic and phenotypic correlation for clustered out of scanner behavioral measures: Left column represent genetic correlation between out of scanner behavioral measures and brain subtypes weights. Right column represent phenotypic correlation between out of scanner behavioral measures and brain subtypes weights. 